\title{
High Throughput Disk Scheduling with Fair Bandwidth Distribution
}

\author{
Paolo Valente, Fabio Checconi
}

\begin{abstract}
Mainstream applications-such as file copy/transfer, Web, DBMS, or video streaming-typically issue synchronous disk requests. As shown in this paper, this fact may cause workconserving schedulers to fail both to enforce guarantees and to provide a high disk throughput. A high throughput can be however recovered by just idling the disk for a short time interval after the completion of each request. In contrast, guarantees may still be violated by existing timestamp-based schedulers, because of the rules they use to tag requests.

Budget Fair Queueing (BFQ), the new disk scheduler presented in this paper, is an example of how disk idling, combined with proper back-shifting of request timestamps, may allow a timestamp-based disk scheduler to preserve both guarantees and a high throughput. Under BFQ each application is always guaranteed-over any time interval and independently of whether it issues synchronous requests-a bounded lag with respect to its reserved fraction of the total number of bytes transferred by the disk device.

We show the single-disk performance of our implementation of BFQ in the Linux kernel through experiments with real and emulated mainstream applications.
\end{abstract} vice.

Index Terms-Scheduling, secondary storage, quality of ser-

\section{INTRODUCTION}

$\mathbf{M}$ OST mainstream applications, such as file transfer, Web, DBMS, Video on Demand or Internet TV, require moving data to/from disk devices. Meeting the disk bandwidth and perrequest delay requirements of these applications and at the same time achieving a high throughput is not an easy task. The first problem is that the time needed to serve a request once dispatched to the disk device, hereafter called service time, is highly variable. Causes are seek and rotational latencies, variation of the transfer rate with the sector position, caching ${ }^{1}$. In addition, few if any of the existing controllers export these physical parameters, which makes it difficult to predict service times based on request positions on the disk. Finally, most mainstream applications usually issue one request or batch of requests at a time. Especially, they block until the only outstanding request/batch has been completed. We denote this type of request or batch of requests as synchronous, for it can be issued only after the outstanding batch/request has been completed. The peculiar arrival pattern of synchronous requests may cause guarantee and disk throughput problems.

Guarantee violations may occur with timestamp-based schedulers, i.e., schedulers that timestamp requests as a function of their arrival time and in essence dispatch them to the disk in ascending timestamp order. The root of the problem is that the arrival of a synchronous request may be arbitrarily delayed by a scheduler

P. Valente is with the Dep. of Computer Engineering of Modena, Italy. F. Checconi is with the Scuola Superiore Sant'Anna, Pisa, Italy.

${ }^{1}$ Other less influential sources of variability are sector sparing and dynamic variation of the disk parameters due to thermal variations. by just delaying the dispatching of the preceding request. Then a delayed synchronous request may get a higher timestamp with respect to the one it would have got if not delayed. This higher timestamp may finally let the request wait for the service of more requests before being dispatched to the disk. Unfortunately, delaying the service and hence the completion of the request will delay the arrival of the successive synchronous request of the same application, and so on. In the end, by just delaying the service of its requests, the scheduler may force the application to issue requests at a deceptively lower rate. If this anomaly occurs, the scheduler just fails to guarantee the reserved bandwidth or the assigned request completion times to the application (even to a greedy one).

In addition, a minimum amount of time is needed for an application to handle a completed request and to submit the next synchronous one. On one hand, this fact may further contribute to violating guarantees with timestamp-based schedulers. On the other hand, it may also prevent a work-conserving scheduler from achieving a high disk throughput. From the disk device standpoint, an application is deceptively idle until it issues the next synchronous request [1]. During one such idle time, the disk head may be moved away from the current position by a workconserving scheduler, thus losing the chance of a close access for applications performing mostly sequential IO. The problem is mitigated by the fact that operating systems typically perform read-ahead for request patterns deemed sequential.

Over-provisioning would be a way to easily guarantee a predictable and short request service time without dealing with the above problems. Unfortunately it entails high purchase, powering and cooling costs [2], and purposely wastes disk bandwidth. In fact, the only option to improve the disk utilization and meet the requirements of many competing applications is properly scheduling disk requests. Several schedulers-such as SCAN (Elevator), C-SCAN, LOOK or C-LOOK [3]-have been defined to achieve the first goal. These algorithms are often implemented also inside modern disk devices, which can internally queue requests and service them in the best order to boost the throughput. Finally, to achieve a high throughput also in presence of deceptive idleness, most Linux standard disk schedulers extend these policies with disk idling: they do not dispatch any other request to the disk for a short time interval (in the order of the seek and rotational latencies) after a synchronous request has been completed. By doing so they give a chance to the (possible) next request of the same application to arrive before the disk arm is moved away. The Anticipatory (AS) disk scheduler [1], extends C-LOOK in this sense.

However, to meet the requirements of most types of applications, guarantees must also be provided on bandwidth distribution or request completion times. In this respect, the problem of algorithms aimed only at maximising the disk throughput is that in the worst case they may delay the service of a request until the whole 
disk has been read or written (age-based policies, as in AS, can be added to mitigate the problem). In contrast, many schedulers, as e.g., SCAN-EDF [4], SATF-DAS [5], JIT [6], Hybrid [7], YFQ [8], pClock [9], adaptive DRR [10], CFQ [11] and adaptations of SFQ [12] have been proposed to provide control on request completion time and/or on bandwidth distribution, while at the same time trying to keep the throughput high.

Apart from CFQ, all of these schedulers are work-conserving and none of them takes the delayed arrival problem into account. Hence, as previously discussed, they may provide a low disk throughput and, if timestamp-based, may violate guarantees. For similar reasons, guarantees for synchronous requests are violated with any scheduler if the disk device performs internal queueing, as discussed in more detail in Section IV. Our experimental results with a single disk thoroughly confirm the expected loss of guarantees for all the analyzed work-conserving timestampbased schedulers, as well as a loss of disk throughput in case of applications performing mostly sequential accesses. Of course, on the opposite end, adopting a naïve disk idling approach in a multidisk system, i.e., idling the entire array of disks on the completion of each sequential synchronous request, may cause throughput loss $[13]^{2}$. Some more effective strategies are mentioned together with our proposal in the next subsection.

In contrast, a non timestamp-based scheduler should not suffer from either problems, provided that some mechanism is adopted to handle deceptive idleness. This is the case for round robin schedulers, as, e.g., CFQ, which does perform disk idling. Unfortunately, as discussed in Section IV and experimentally shown in Section V, they exhibit a higher delay/jitter in request completion times than the timestamp-based scheduler we propose.

\section{A. Proposed Solution}

In this paper we propose a proportional share timestampbased disk scheduler, called Budget Fair Queueing (BFQ). As in any proportional share scheduler, in BFQ each application is guaranteed its reserved fraction (share) of the disk throughput, irrespective of its behaviour. Hence neither the request arrival pattern of the application needs to be known or the application itself needs to be modified to provide such a guarantee.

BFQ serves applications as follows. First, when enqueued, an application is assigned a budget, measured in number of sectors to transfer. Once selected, the application gets exclusive access to the disk. During the service of the application disk idling is performed to wait for the arrival of synchronous requests (only if these requests are deemed sequential, see Subsection II-B). Finally, if the application runs out of either its backlog or its budget, it is deselected and assigned a new budget. This service scheme allows BFQ to achieve a high throughput if applications issue mostly sequential requests. In contrast, for random workloads it is not as optimal as a global policy, like, e.g., C-LOOK. It is however worth noting that with random workloads even an optimal policy can achieve only a small fraction of the disk rate. In fact, where possible, caches are usually tuned so as to reduce disk access and achieve feasible response times.

Applications are scheduled by the internal Budget- $\mathrm{WF}^{2} \mathrm{Q}+(\mathrm{B}-$ $\mathrm{WF}^{2} \mathrm{Q}+$ ) scheduler as a function of their budgets. The latter is

\footnotetext{
${ }^{2}$ In [13] an extensive comparative analysis of the performance of several disk schedulers is reported, in terms of aggregate throughput, with different workloads and also in presence of internal queueing and multiple disks.
}

a slightly extended version of $\mathrm{WF}^{2} \mathrm{Q}+[14]$, a proportional share (fair-queuing) packet scheduler. $\mathrm{B}-\mathrm{WF}^{2} \mathrm{Q}+$ basically differs from the original packet scheduler in that, first, it handles the case where an application becomes idle before consuming all of its budget, and, second, it properly shifts backwards timestamps to conceal delayed arrivals. Thanks to this characteristics and to the fact that it works in the service and not in the time domain, BFQ provides the following guarantee to each application, over any time interval, with any workload and regardless of the disk physical parameters: each application is guaranteed the minimum possible lag, achievable by serving applications budget-by-budget, with respect to the minimum amount of service, measured in number of sectors transferred, that the application should receive according to its reserved share of the disk throughput and to the total amount of service provided by the system. A loose upper bound to this lag is $3 B_{\max }$, where $B_{\max }$ is the maximum budget that can be assigned to any application. In general, BFQ can be fine-tuned to achieve the desired trade-off between throughput boosting and maximum per-application lag, by tuning $B_{\max }$ and a few other configuration parameters. In addition, a simplified interface is provided for users not concerned with low-level details.

The above sector guarantees can be turned into time guarantees as follows. First, the aggregate throughput achieved with the desired values of the configuration parameters must be measured for the expected worst-case request pattern (see Section III-D). Then, worst-case time guarantees can be computed as a function of the aggregate throughput with a simple closed-form expression. Note that, on one side no other disk physical parameter needs to be known or measured, whereas, on the other side, there is no possibility with any scheduler to provide practical time guarantees without knowing at some degree at least the locality of the expected request pattern.

With regard to computational cost and implementation issues, BFQ is defined without using disk physical parameters, and has $O(\log N)$ worst-case cost, in the number of competing applications, per request insertion or dispatch (this cost boils down to $O(1)$ if approximate implementations [15] of $\mathrm{B}-\mathrm{WF}^{2} \mathrm{Q}+$ are adopted). We have implemented BFQ in the Linux kernel (currently for 2.6.21-29 kernels [16]) and experimentally evaluated its single-disk performance with file transfer, Web server, DBMS and video streaming applications (Section V). In general, the main contribution of this paper is showing through BFQ a way to combine disk idling and timestamp back-shifting to preserve both guarantees and a high throughput in presence of synchronous requests. This technique may be applied also to other timestampbased schedulers. Finally, to preserve a high throughput in a multiple-disk system, the disk idling scheme described so far should be extended to allow, e.g., one outstanding request per disk. Or alternatively a hierarchical approach might be adopted, leaving the disk idling task only to the local per-disk schedulers. Carefully investigating these solutions is out of the scope of this paper.

\section{B. Organization of the Paper}

In Section II we describe BFQ and report its computational cost, whereas in Section III we show its service properties in the sector and time domains. In Section IV we provide a brief survey of related work. Finally, in Section V we compare the 


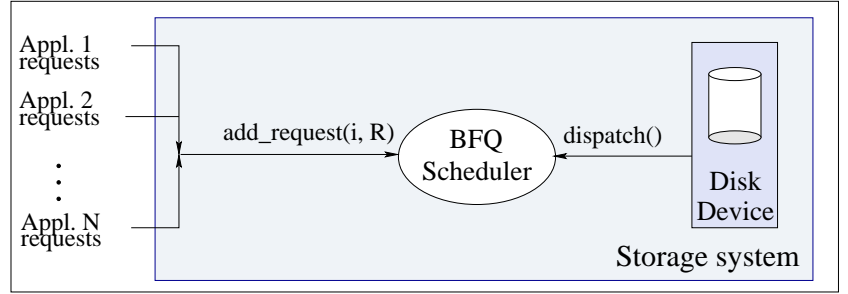

Fig. 1. System model.

TABLE I

DEFINITIONS.

\begin{tabular}{l|l}
\hline Symbol & Meaning \\
\hline \hline$R_{i}^{j}$ & $j$-th request issued by the $i$-th application \\
\hline$L_{i}^{j}$ & Size of $R_{i}^{j}$ \\
\hline$L_{\max }$ & $\max _{i, j} L_{i}^{j}$ \\
\hline$a_{i}^{j}, s_{i}^{j}, c_{i}^{j}$ & Arrival, start and completion time of $R_{i}^{j}$ \\
\hline$W_{i}(t)$ & Amount of service received by the $i$-th application \\
\hline$W(t)$ & Total amount of service delivered by the system \\
\hline$T_{w a i t}$ & Time waited before deeming an application as idle \\
\hline$B_{i, \max }$ & Maximum budget assigned to the $i$-th application \\
\hline$B_{\max }$ & max $B_{i, \max }$ \\
\hline$f\left(t_{1}, t_{2}\right)$ & $f\left(t_{2}\right)-f\left(t_{1}\right)$ \\
\hline$\phi_{i}$ & Weight of the $i$-th application \\
\hline$T_{F I F O}$ & $\begin{array}{l}\text { Queueing time after which (queued) requests must be served } \\
\text { in FIFO order }\end{array}$ \\
\hline
\end{tabular}

performance of BFQ against several research and production schedulers.

\section{BFQ}

After defining the system model in the next subsection, we introduce the logical scheme and the main algorithm in the successive two subsections, where $\mathrm{B}-\mathrm{WF}^{2} \mathrm{Q}+$ is used as a black box providing application enqueue/dequeue operations. We then describe $\mathrm{B}-\mathrm{WF}^{2} \mathrm{Q}+$ in detail in Subsections II-D and II-E.

\section{A. System model}

We consider a storage system composed of a disk device and the BFQ scheduler, as in Fig. 1. The former contains a disk, which we model as a sequence of contiguous fixed size sectors, each identified by its position in the sequence. The disk device services two types of disk requests, respectively the reading and the writing of a set of contiguous sectors. After receiving the start command or completing a request, the disk device asks for the next request to serve by invoking the function dispatch exported by the BFQ scheduler.

At the opposite end, requests are issued by the $N$ applications served by the storage system (applications here stand for the possible entities that can compete for disk access in a real system, as, e.g., threads or processes). The meaning of the notations hereafter introduced is also summarized in Table I. The $i$-th application issues its $j$-th request $R_{i}^{j}$ by invoking the function add_request exported by the scheduler, and passing the index $i$ and the request $R_{i}^{j}$. We define as size $L_{i}^{j}$ of $R_{i}^{j}$ the number of sectors to read or write, and as position/end of $R_{i}^{j}$ the position of the first/last of these sectors. We say that two requests are sequential if the position of the second request is just after the end of the first one. We define as arrival, start and completion

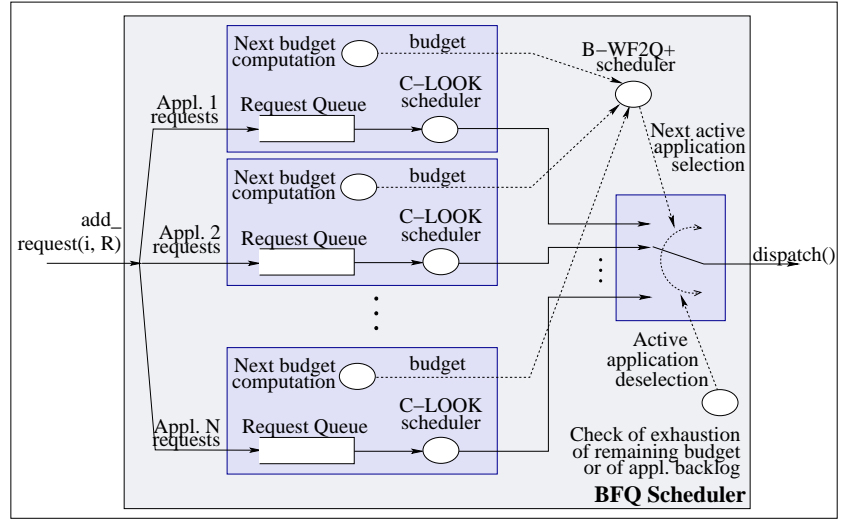

Fig. 2. BFQ Logical Scheme.

time of a request the time instants $a_{i}^{j}, s_{i}^{j}$ and $c_{i}^{j}$ at which the request $R_{i}^{j}$ is issued by the $i$-th application, starts to be served and is completely served by the disk device, respectively. We say that a request is synchronous if it can be issued by an application only after the completion of its previous request. Otherwise the request is denoted as asynchronous.

We say that an application is receiving service from the storage system if one of its requests is currently being served. Both the amount of service $W_{i}(t)$ received by an application and the total amount of service $W(t)$ delivered by the storage system are measured in number of sectors transferred during $[0, t]$. For each application $i$, we let $B_{i, \max }$ denote the dynamically configurable maximum budget, in number of sectors, that BFQ can assign to it. We define $B_{\max } \equiv \max _{i} B_{i, \max }$.

We say that an application is backlogged if it has pending requests. In addition, to deal with the delayed arrival and the deceptive idleness problems an application is denoted as quasibacklogged at time $t$ if either it is backlogged, or it is not backlogged but its backlog emptied not before time $t-T_{\text {wait }}$, where $T_{\text {wait }}$ is a system-wide dynamically configurable time interval. Otherwise the application is deemed as idle. Moreover we say that an application $i$ enjoys the short-or-independent arrival property (SI property for short) if, for each request $R_{i}^{j}$, either $R_{i}^{j}$ is asynchronous, or $a_{i}^{j}-c_{i}^{j-1} \leq T_{\text {wait }}$. The actual adherence of real-world applications to the SI property is discussed in Section III, whereas hereafter we assume that applications do enjoy this property.

Each application has a fixed weight $\phi_{i}$ assigned to it. Without losing generality, we assume that $\sum_{q=1}^{N} \phi_{q} \leq 1$. Given a generic function of time $f(t)$, we define $f\left(t_{1}^{-}\right) \equiv \lim _{t \rightarrow t_{1}^{-}} f(t)$ and $f\left(t_{1}, t_{2}\right) \equiv f\left(t_{2}\right)-f\left(t_{1}\right)$. Finally, given any time interval $\left[t_{1}, t_{2}\right]$ during which the $i$-th application is continuously quasibacklogged, we define its reserved service during $\left[t_{1}, t_{2}\right]$ as $\phi_{i} \cdot W\left(t_{1}, t_{2}\right)$. Suppose that $R_{i}^{j+1}$ is a synchronous request, and let $\bar{c}_{i}^{j}$ be the time instant at which the request $R_{i}^{j}$ would be completed if the $i$-th application received exactly its reserved service during $\left[a_{i}^{j}, \bar{c}_{i}^{j}\right]$. We say that the arrival of $R_{i}^{j+1}$ is (deceptively) delayed if $c_{i}^{j}>\bar{c}_{i}^{j}$.

\section{B. Logical scheme}

The logical scheme of BFQ is depicted in Fig. 2. Differently from Fig. 1, here solid arrows represent the paths followed by requests until they reach the disk device. On the contrary, dashed 
arrows represent flows of information or internal commands. Finally, circles represent algorithms or operations. There is a request queue for each application. Requests are inserted into the right queue by the add_request function.

At any time each application has a budget assigned to it, measured in number of sectors. Let $B_{i}^{l}$ be the $l$-th budget assigned to the $i$-th application. At system start-up, all applications are assigned the same default budget $B_{i}^{0}$. Disk access is granted to one application at a time, denoted as the active application. When the new active application is selected, its current budget is assigned to a special remaining budget counter. Each time a request of the active application is dispatched, the remaining budget counter is decreased by the size of the request. Moreover, if the request queue gets empty at time $t$, a timer is set to $t+T_{\text {wait }}$ to wait for the possible arrival of the next request. This may leave the disk idle, but prevents BFQ from switching to a different application if the active application is deceptively idle and enjoys the SI property. In addition to not breaking a possible sequence of close or sequential accesses, this waiting is also instrumental in concealing delayed arrivals, as shown in Subsection II-E. However, waiting for the arrival of non-sequential requests provides no benefit. Hence, as CFQ, BFQ automatically reduces $T_{\text {wait }}$ to a very low (configurable) value for applications performing random IO (currently $2 \mathrm{~ms}$, see the code for details [16]). If the active application issues no new request before the timer expiration, it is deemed idle. The active application is exclusively served until either there is not enough remaining budget to serve the next request, or the application becomes idle. At this point the next budget $B_{i}^{l+1}$ of the application is computed. Basically, $B_{i}^{l+1}$ is obtained by increasing or decreasing $B_{i}^{l}$, depending on whether the application consumed all of its budget or ran out of its backlog before consuming it. The next active application is then chosen by $\mathrm{B}-\mathrm{WF}^{2} \mathrm{Q}+$, which schedules enqueued applications as a function of their budgets (see Subsection II-E).

To guarantee a controllable per-application maximum queueing time, the order in which the requests are extracted from the queue of the active application depends on a user-configurable $T_{F I F O}$ parameter. When the next request of the $i$-th application is to be dispatched at time $t$, if $a_{i}^{j}+T_{F I F O}>t$ holds for all the queued requests $R_{i}^{j}$, then the next request is chosen in C-LOOK order [3], otherwise the oldest request is picked.

\section{Main algorithm}

The main BFQ algorithm is shown in Fig. 3 using pseudocode. The function add_request first inserts the new request $R$ in the application queue, then, if there are more than one request, nothing is to be done. Otherwise, if the application is not the active one, the application is enqueued in the $\mathrm{B}-\mathrm{WF}^{2} \mathrm{Q}+$ scheduler. If the (active) application is waiting for the arrival of the next request, the timer is unset.

The function dispatch returns a no request indication if all applications are idle or if the active application is waiting for the arrival of the next request (disk idling). On the contrary, if the active application has not enough remaining budget for its next request, the application is deselected (lines 24-38). Moreover, through the call to the function b-wf $2 q+$-update_vfintime (described in the next subsection) at lines 27-28, the application timestamps are updated to account only for the service received. The fact that the application did not empty all of its backlog is assumed as an indication that it issues batches of requests with a larger cumulative size than the last assigned budget. Hence, the application budget is increased by a configurable quantity, provided that the resulting value is not higher than $B_{i, \max }$ (lines 30-34). Finally, the application is enqueued into $\mathrm{B}-\mathrm{WF}^{2} \mathrm{Q}+$ with its new budget (line 36). Then, if no application is active (lines 39-43), the next active application is picked (and removed) from the $\mathrm{B}-\mathrm{WF}^{2} \mathrm{Q}+$ scheduler. The next request $R$ to serve is extracted from the queue of the active application and the remaining budget counter is decremented (lines 44-49).

Finally, if the queue of the active application becomes empty, the timer is set to the current time plus $T_{\text {wait }}$ (lines 50-51). If no new request is issued by the application before timer expiration, the function timer_expiration (lines 59-66) gets called: the application is declared idle, and a new budget equal to the previously consumed one is assigned to it. Finally, the timestamps of the application are properly updated to account only for the actual amount of service it received (see the next subsection).

The adopted simple linear increase/instantaneous decrease of budgets is of course only one of the possible options. It showed good performance in terms of both aggregate throughput and short term guarantees in our experiments. For simplicity, many low-level details have been omitted here, as the fact that disk idling is obviously performed only for synchronous requests. The interested reader is referred to [16]. Before leaving this subsection, it is worth noting that BFQ actually provides a flexible framework, in that both the $\mathrm{B}-\mathrm{WF}^{2} \mathrm{Q}+$ and $\mathrm{C}-\mathrm{LOOK}$ schedulers can be replaced with different schedulers (Fig. 2). Hence, different trade-offs among type of guarantees, computational cost and throughput boosting degree can be achieved.

\section{Disk Weighted Fair Queueing}

In this subsection we provide a brief survey of the main concepts behind the original $\mathrm{WF}^{2} \mathrm{Q}+$ algorithm (see [14] and [17] for details), (re)formulated in the disk scheduling domain. These concepts are the basis for $\mathrm{B}-\mathrm{WF}^{2} \mathrm{Q}+$, which is then described in detail in the next subsection.

Hereafter we use the term batch to denote the set of the requests served using a given budget. We define a batch as pending at time $t$ if it has not yet been completely served at time $t$ (each application has at most one pending batch at a time). We define two systems as corresponding if they serve the same applications and, at any time instant, they provide the same total amount of service per time unit.

$\mathrm{WF}^{2} \mathrm{Q}+$ approximates on a packet-by-packet basis the ideal service provided by a work-conserving packet-based fluid system. In $\mathrm{B}-\mathrm{WF}^{2} \mathrm{Q}+$ we map the concept of packet into the concept of batch: $\mathrm{B}-\mathrm{WF}^{2} \mathrm{Q}+$ approximates on a batch-by-batch basis the service provided by a corresponding fluid system that may serve more than one application at a time. Hereafter this system is called just the ideal system, as opposed to the real system, i.e., to the storage system in Fig. 1. The ideal system is used as a reference because it distributes the total service among applications so as to guarantee to each application a bounded lag, over any time interval, with respect to its reserved service (see the appendix for details). Moreover, in the ideal system, each request is completed no later than the time instant at which it has to be completed according to the reserved service of the issuing application. On the contrary, as shown in the Section III, this does not hold in the real system. Hence a synchronous request in the real system may 


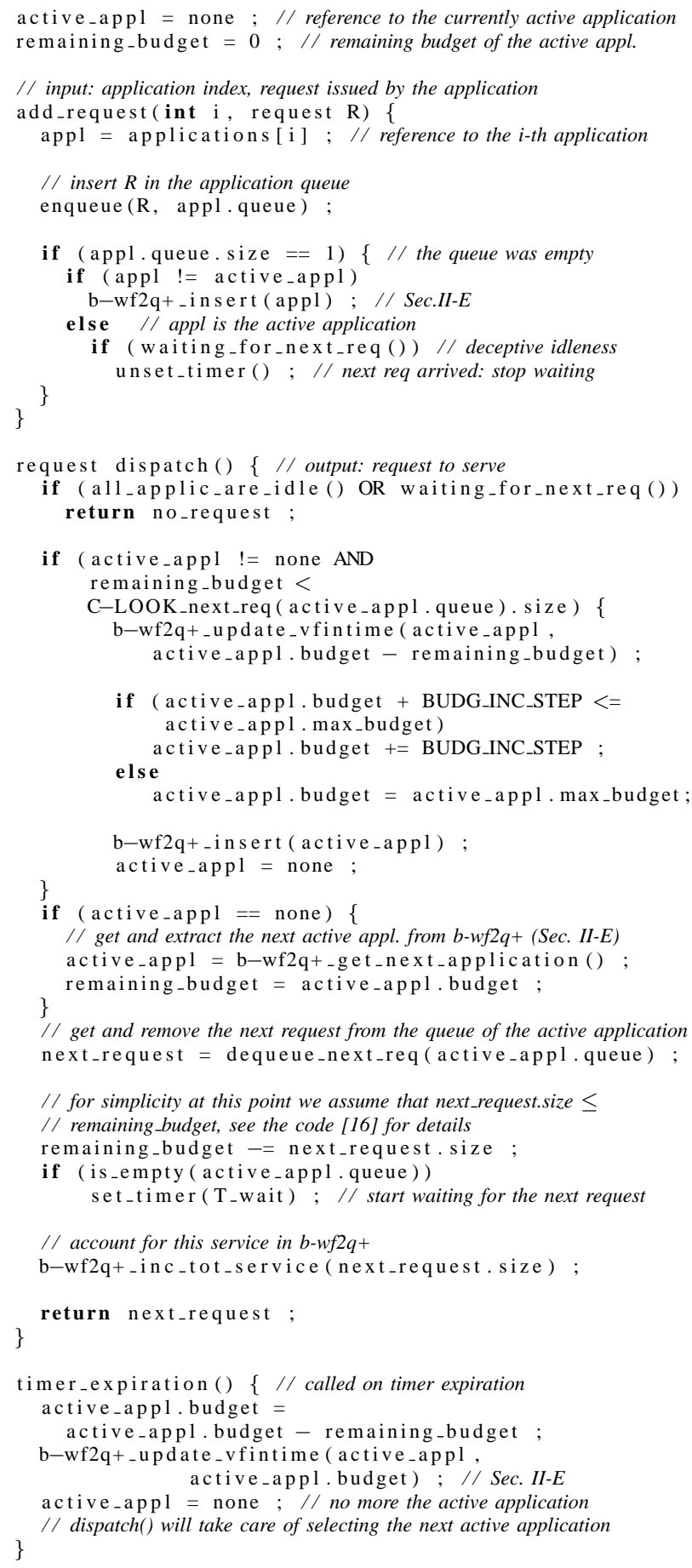

Fig. 3. BFQ Main Algorithm

arrive later than in the ideal system. This fact may cause request arrivals to be deceptively delayed in the real system.

An application is eligible at time $t$ if its pending batch would have already started to be served in the ideal system at time $t$. $\mathrm{B}-\mathrm{WF}^{2} \mathrm{Q}+$ tries to complete the service of application batches in the same order as the ideal system. Moreover, it chooses the next application to serve only among the eligible ones.

This policy is efficiently implemented by timestamping each application with the values assumed by a special application virtual time function at the start and completion times of its pending batch in the ideal system. These two values are called virtual start and finish time, $S_{i}(t)$ and $F_{i}(t)$, of the application or, equivalently, of its pending batch at time $t$. They are computed as a function of a common system virtual time function $V(t)$ when an application is (re)inserted into the scheduler (see [14] or [17]). Although it seems a contradiction in terms, the unit of measure of the virtual time is the service (see the appendix for details). Especially the virtual time of each backlogged application grows as the amount of service received by the application, divided by its weight.

\section{E. $B-W F^{2} Q+$ and timestamp back-shifting}

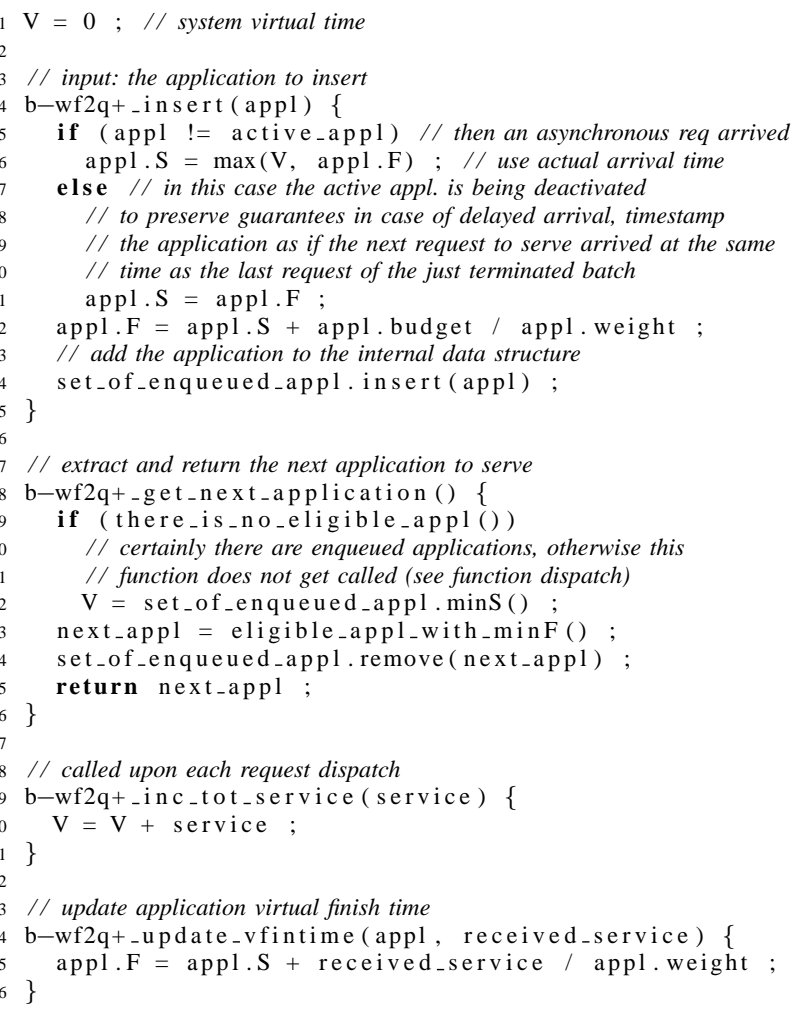

Fig. 4. $\quad \mathrm{B}-\mathrm{WF}^{2} \mathrm{Q}+$.

The $\mathrm{B}-\mathrm{WF}^{2} \mathrm{Q}+$ algorithm is shown in Fig. 4 using pseudocode. An application is inserted into the scheduler by calling the function $b-w f 2 q+$-insert. If the application is not the active one, then, since $T_{\text {wait }}$ is waited for before deactivating an application and applications enjoy the SI property, $b-w f 2 q+$ insert is called as a consequence of the arrival of an asynchronous request. In this case the virtual start and finish times of the application are computed as a function of the actual time at which $\mathrm{b}-\mathrm{wf} 2 \mathrm{q}+$-insert is called, i.e., the actual request arrival time, using the same formulas as in $\mathrm{WF}^{2} \mathrm{Q}+[14]$.

On the other hand, if the application to insert is the active one, then according to Fig. 3 line 36, the application is necessarily being enqueued into $\mathrm{B}-\mathrm{WF}^{2} \mathrm{Q}+$ after a deactivation and not because of the arrival of a request. Let $R_{i}^{j}$ be the next request to serve of the application (there is certainly one). As explained below in detail, after a deactivation $F_{i}$ is assigned the value assumed by the application virtual time upon the completion of the last served request, in this case $R_{i}^{j-1}$, in the ideal system 
(Fig.3 lines 27-28). Let $\overline{F_{i}}$ be this value. According to [14], the exact value of the application virtual time upon the arrival of $R_{i}^{j}$ should have been equal to $\max \left(\overline{F_{i}}, V\left(a_{i}^{j}\right)\right)$. On the contrary, in B$\mathrm{WF}^{2} \mathrm{Q}+\overline{F_{i}}$ is unconditionally assigned to $S_{i}$, as if $R_{i}^{j}$ had arrived at a time instant between the arrival time $a_{i}^{j-1}$ and the completion time in the ideal system of the previous request $R_{i}^{j-1}$. This fictitious backward shift conceals the possibly delayed arrival of $R_{i}^{j}$ and of the successive requests served using the same budget as $R_{i}^{j}$. Moreover, the worst-case guarantees of the other applications are not endangered, as the guarantees provided to each application are independent of the arrival time of the requests of the other applications.

The function b-wf2q+_get_next_application returns the eligible application with the minimum virtual finish time and removes it from the internal data structure (lines 23-25). In case there are quasi-backlogged applications, but no one is eligible (lines 19-22), $V(t)$ is pushed-up to the minimum virtual start time among all the quasi-backlogged applications (the latter coincide with the applications enqueued in $\mathrm{B}-\mathrm{WF}^{2} \mathrm{Q}+$ when $\mathrm{b}-\mathrm{wf} 2 \mathrm{q}+$-get_next_application is invoked). Since an application is eligible if and only if its virtual start time is not higher than the system virtual time, this jump guarantees $\mathrm{B}-\mathrm{WF}^{2} \mathrm{Q}+$ to be work-conserving [14]. However, as shown in Subsection II-B the overall BFQ algorithm is not work-conserving, as it waits for the arrival of a new request before serving the next one.

Pushing up the system virtual time is a delicate operation with respect to the fictitious backward shift of arrival times. Let the $i$-th application be one of the applications that are idle in the real system at time $\bar{t}$ when a jump is performed. Suppose for a moment that a delayed synchronous request $R_{i}^{j}$ arrives after time $\bar{t}$. Since the $i$-th application is not taken into account in computing $V(\bar{t})$, the jump would be conceptually incompatible with a fictitious backward shift of the arrival of $R_{i}^{j}$ to (or before) time $\bar{t}$. Hence it is easy to show that it would not be possible to conceal this delayed arrival without violating the guarantees of the $i$-th application. Fortunately, if the $i$-th application enjoys the SI property, it is not possible that $a_{i}^{j}>\bar{t}$, because $T_{w a i t}$ seconds are waited for before invoking b-wf2q+_get_next_application.

$V(t)$ is also incremented by the size of the just dispatched request upon each request dispatch (function b-wf2q+_inc_tot_service at lines 29-31). For a perfect tracking of the ideal system, $V(t)$ should be continuously increased by the amount of service provided by the disk device. Of course, this is impossible in a real system, because the disk device does not export continuous information on the amount of service provided. Suppose that an idle application issues a new request $R$ while a request $\bar{R}$ is under service, and let $\bar{c}$ be the completion time of $\bar{R}$. Due to the step-wise increment of $V(t)$, the application may be timestamped as if $R$ actually arrived at time $\bar{c}$. Of course, since the application is enqueued before time $\bar{t}$, the worst-case effect of this wrong time-stamping is delaying the service of $R$ as if it arrived at time $\bar{t}$. The consequences of this fact on the service guarantees are shown in Section III.

The last important difference between $\mathrm{B}-\mathrm{WF}^{2} \mathrm{Q}+$ and $\mathrm{WF}^{2} \mathrm{Q}+$ is that the latter also handles the fact that an application may not use all of its budget. This possibility affects only time guarantees, as shown in Subsection III-B. Here we highlight just that, before an application that did not use all of its budget may be enqueued again, its virtual finish time is properly updated by calling b-wf2q+_update_vfintime (lines 34-36) to account only for the actual service received.

Finally, B-WF ${ }^{2} \mathrm{Q}+$ can be implemented at $O(\log N)$ or $O(1)$ cost per application insertion/extraction [15], depending on whether exact or approximate timestamps are used. Since all the other operations in Fig. 3 have $O(1)$ cost, the overall BFQ scheduler can be implemented at $O(\log N)$ or $O(1)$ cost per request insertion/extraction.

The basic algorithm reported in this subsection does not contain many of the details of the complete version. The latter, e.g., autonomously adapts to a dynamic application set and, to allow the users to choose the weights in a simpler and more flexible way, it poses no constraint on the values of the weights [18]. Of course in this case it may happen that $\Phi_{T O T} \equiv \sum_{i=1}^{N} \phi_{i}>1$, but the service properties of BFQ still hold after replacing $\phi_{i}$ with $\frac{\phi_{i}}{\Phi_{T O T}}$ in the following inequalities.

\section{SERVICE PROPERTIES}

In this section we report the service properties of $\mathrm{BFQ}$, in both sector (bandwidth distribution) and time domains. We also show how to perform admission control and to provide time guarantees. Finally we show how to achieve the desired trade-off between fairness granularity and throughput boosting.

Before proceeding, it is important to identify the set of applications for which the service properties of BFQ actually hold also in presence of delayed arrivals. From Section II we know that BFQ conceals the delayed arrivals of the requests issued by the applications that meet the SI property. Hence BFQ guarantees to these applications the same amount of service and the same per-request completion time as if the arrival of each of their synchronous requests would not have been delayed. As a consequence, one would set $T_{\text {wait }}$ as high as possible to include as many applications as possible. However, the value of $T_{\text {wait }}$ has an important impact on the disk throughput. It may provide significant boosting in presence of deceptive idleness, but only if its value is in the order of the seek and rotational latencies [1], namely a few milliseconds. In contrast, higher values may cause progressive performance degradation, as the disk may be left idle for too long.

Applications commonly alternate phases during which they make intense use of the disk device and phases during which they rarely access it. Fortunately, even for the above mentioned beneficial low value of $T_{\text {wait }}$, during the former phases the SI property holds for the majority of mainstream applications. On the other hand, should an application not meet the SI property in the other phases, the possible degradation of the guarantees on bandwidth distribution and request completion times would have a negligible impact on the overall application performance.

\section{A. Sector-domain properties}

To show BFQ sector guarantees, we refer to a sector-variant of the Bit-Worst-case Fair Index (Bit-WFI), originally defined in packet systems [14]. This index, which we denote as Sector-WFI, allows us to predict the minimum amount of service guaranteed by a system to an application over any time interval during which the application is continuously quasi-backlogged (Section II). The following theorem holds.

Theorem 1: For any time interval $\left[t_{1}, t_{2}\right]$ during which the $i$ th application is continuously quasi-backlogged, BFQ guarantees that:

$\phi_{i} \cdot W\left(t_{1}, t_{2}\right)-W_{i}\left(t_{1}, t_{2}\right) \leq B_{\max }+B_{i, \max }+L_{\max }$. 
The right hand side in (1) is the Sector-WFI of BFQ. Note that, if an application enjoys the SI property, then the time intervals during which it needs to access the disk safely coincide with the time intervals during which it is quasi-backlogged. Given the strong similarities between $\mathrm{WF}^{2} \mathrm{Q}+$ and $\mathrm{BFQ}$, the proof of Theorem 1 is basically an extension of the proof of the Bit-WFI of $\mathrm{WF}^{2} \mathrm{Q}+[14]$. Whereas the full proof is reported in the appendix, an intuitive justification of each component of the bound follows.

The component $B_{\max }$ measures the deviation from the ideal service due to not respecting the batch completion order of the ideal system. More precisely, if the $i$-th applications has a lower virtual finish time than the active one, but becomes backlogged too late, it may unjustly wait for the service of at most $B_{\max }$ sectors before accessing the disk.

The second component, $B_{i, \max }$, stems from the fact that, if there is no constraint on the request arrival pattern, BFQ guarantees the real system to be in advance in serving the $i$ th application for at most $B_{i, \max }$ sectors with respect to the minimum guaranteed service at time $t_{1}$. The application may pay back for this extra service during $\left[t_{1}, t_{2}\right]$. However, it is worth noting that this may happen only if a request $R_{i}^{j}$ may arrive before the maximum completion time guaranteed in the ideal system to the previous request $R_{i}^{j-1}$. Hence, on the opposite end, if this never occurs, i.e., the application never asks for more than its reserved service, the component $B_{i, \max }$ is not present at all.

The last term follows from the stepwise approximation of $V(t)$. Basically, due to wrong time-stamping, requests may be erroneously treated as if arrived, with respect to the actual arrival time, after a time interval during which the ideal system may have served at most $L_{\max }$ sectors.

It is important to note that the rightmost term in (1) does not grow with the time or the (total) amount of service, hence the long term bandwidth distribution is unconditionally guaranteed. Furthermore, (1) provides a simple relationship between the shortterm bandwidth distribution and the value of the parameters that influence the aggregate disk throughput, as detailed in Subsection III-D. Finally, it is easy to prove that no scheduler that exclusively serves applications batch-by-batch may guarantee a lower Sector-WFI than BFQ. Hence BFQ provides optimal worst-case bandwidth distribution guarantees among this class of schedulers.

\section{B. Time-domain properties}

The following theorem is the starting point for computing the time guarantees of BFQ. Let $t_{1} \leq a_{i}^{j}$ be a generic time instant such that the $i$-th application is continuously quasi-backlogged during $\left[t_{1}, c_{i}^{j}\right]$. To compute a worst-case upper bound to $c_{i}^{j}$, we assume that all the applications, except for the $i$-th one, ideally start to issue asynchronous requests back-to-back from time $t_{1}$ (i.e., without waiting for the completion of their outstanding requests). Moreover, to prevent BFQ from increasing budgets and hence boosting the throughput more than it would happen in the actual scenario, we assume that the maximum value of the budget of each application, except for the $i$-th application, is set to its average value in the real scenario. Finally, thanks to the fact that BFQ conceals delayed arrivals for applications enjoying the SI property, if $R_{i}^{j}$ is a delayed synchronous request but the application does enjoy the SI property, in the following theorem $a_{i}^{j}$ can be safely assumed to be equal to the time instant at which $R_{i}^{j}$ would have arrived if it had not been delayed.
Theorem 2: Given a request $R_{i}^{j}$, let $t_{1} \leq a_{i}^{j}$ be a generic time instant such that the $i$-th application is continuously quasibacklogged during $\left[t_{1}, c_{i}^{j}\right]$. Let $T_{a g g}$ be the minimum aggregate disk throughput during an interval $\left[t_{1}, c_{i}^{j}\right]$ under the above worstcase assumptions. Finally, let $L_{i}^{j}$ be the size of $R_{i}^{j}$ and $A_{i}\left(t_{1}, a_{i}^{j}+\right.$ $\left.T_{F I F O}\right)$ be the sum of the sizes of the requests issued by the $i$ th application during $\left[t_{1}, a_{i}^{j}+T_{F I F O}\right)$ plus $L_{i}^{j}$. The following inequality holds:

$$
\begin{aligned}
c_{i}^{j}-t_{1} & \leq \\
\frac{Q_{i}\left(t_{1}^{-}\right)+A_{i}\left(t_{1}, a_{i}^{j}+T_{F I F O}\right)+\left(B_{i}^{l}-L_{i}^{j}\right)+B_{i, \max }}{\phi_{i} T_{a g g}}+ & \\
+\frac{B_{\max }+B_{i, \max }+L_{\max }}{T_{\text {agg }}} &
\end{aligned}
$$

where $Q_{i}\left(t_{1}^{-}\right)$is the sum of the sizes of the requests of the $i$-th application not yet completed immediately before time $t_{1}$, and $B_{i}^{l}$ is (the size of) the budget assigned to the $i$-th application to serve the batch that $R_{i}^{j}$ belongs to.

As before, the proof of this theorem, which can be found in the appendix, is just an extension of the proof of the Time-WFI of $\mathrm{WF}^{2} \mathrm{Q}+$. We discuss here the terms in (2), and in Subsec. III-C how to use (2) to perform admission control and to provide actual time guarantees.

The right hand side of (2) can be rewritten as $\frac{1}{\phi_{i} T_{a g g}}$. $\left(Q_{i}\left(t_{1}^{-}\right)+A_{i}\left(t_{1}, a_{i}^{j}+T_{F I F O}\right)\right)+d_{i}^{j}$. It is easy to see that the first component represents the worst-case completion time of $R_{i}^{j}$ in an ideal system guaranteeing no lagging behind the reserved service over any time interval. In contrast $d_{i}^{j}$ represents the worstcase delay with respect to the ideal worst-case completion time. With regard to the first component, note that, if the $i$-th application issues only synchronous requests, the latter are always served in FIFO order. This is equivalent to assuming $T_{F I F O}=0$.

The first component of the worst-case delay, $B_{i}^{l}-L_{i}^{j}$, stems from that the batch that $R_{i}^{j}$ belongs to is not timestamped (and scheduled) as a function of $L_{i}^{j}$, but as a function of $B_{i}^{l}$. Hence, in the worst-case the service of $R_{i}^{j}$ may be delayed proportionally to the difference $B_{i}^{l}-L_{i}^{j}$. Finally, the $B_{\max }, B_{i, \max }$ (which appears twice in $d_{i}^{j}$ ) and $L_{\max }$ terms can be explained using the same arguments as for the same terms in (1).

\section{Admission control and time guarantees}

We now discuss how to use (2) for performing admission control and providing actual bandwidth and completion time guarantees. First of all, the aggregate throughput $T_{a g g}$ must be known at some extent. The tricky aspect is that $T_{a g g}$ is in its turn a function of the many user-configurable parameters $B_{i, \max }, B_{\max }, T_{F I F O}, T_{\max }$ and $T_{w a i t}$. However, as shown in detail in Subsection III-D, basing upon (2), the desired tradeoff between completion times and $T_{a g g}$ can be achieved by iteratively tuning the values either of each of these parameters or of just the throughput boosting level parameter. The accuracy of the computed guarantees then depends on how accurately $T_{a g g}$ itself is known (worst-case or average value, variance, confidence interval, ...). In this respect recall that referring to the expected throughput/service time, albeit unavoidably affected by approximations, is the only option to provide practical time guarantees with any disk scheduling algorithm.

Once known the worst-case throughput, the requirements of an application requesting a long term throughput $T_{i}$ and no other type of guarantee can be fulfilled by assigning to it a weight 
$\phi_{i}=\frac{T_{i}}{T_{a g g}}$. The application is then admitted only if the resulting sum of the weights $\sum_{q=1}^{N} \phi_{q}$ is still no higher than 1 .

In contrast, to provide guarantees on single request completion times to an application, the request arrival pattern of the application needs to be modeled too. A general request arrival model is the periodic or sporadic pattern: the application issues requests with a size of at most $Q_{i}$ sectors, and with a period or minimum inter-arrival time of $P_{i}$ seconds. This pattern models soft realtime applications, as, e.g., audio or video streaming ones. Since $T_{a g g} \cdot P_{i}$ sectors are transferred during $P_{i}$, it follows that, to meet the throughput requirements of the application, it is enough to set $\phi_{i}=\frac{Q_{i}}{T_{a g g} \cdot P_{i}}$. As before, for the application to be admitted the resulting sum of the weights must still be no higher than 1 .

With regard to the guarantees on request completion times provided to such a type of applications, it is worth noting that $P_{i}$ coincides with the maximum time needed by the ideal system to complete the last issued request. Hence, at time $a_{i}^{j}, R_{i}^{j}$ has been certainly completed in the ideal system, and, for what is said in the previous two subsections, the $B_{i, \max }$ component is absent from (2). In the end, the application must tolerate a worst-case delay (jitter)

$$
d_{i, \max } \equiv \max _{j} d_{i}^{j} \leq \frac{B_{i, \max }-L_{i, \min }}{\phi_{i} T_{a g g}}+\frac{B_{\max }+L_{\max }}{T_{a g g}},
$$

where $L_{i, \min } \equiv \min _{j} L_{i}^{j}$. Stated in other terms, it is possible to meet the requirements of periodic/sporadic soft real-time applications with relative deadlines equal to $P_{i}+d_{i, \max }$. These requirements do match, e.g., (buffered) video and audio streaming applications.

To show possible values of the bounds (2) and (3) in a real system, and to demonstrate the feasibility of interactive and soft real-time applications with BFQ, a Web, DBMS and Video-onDemand service are considered in Section V.

\section{Throughput Boosting}

Larger budgets increase the probability of serving larger bursts of close or even sequential requests, and hence of achieving a higher throughput. Besides, a large value of $T_{F I F O}$ may boost the throughput in presence of asynchronous requests. In this respect, also recall that most mainstream applications issue only synchronous requests. Hence, in a system serving this kind of applications $T_{F I F O}$ has no impact either on the throughput or on the time guarantees (Subsection III-B). In contrast $T_{\text {wait }}$ may be just set to the most effective value for the target disk device, equal to the device-dependent average cost of seek and rotational latencies, usually between 4 and $8 \mathrm{~ms}$ (and set by default to $4 \mathrm{~ms}$ in the current release of BFQ).

BFQ exports a last low-level configuration parameter related to disk throughput, namely the system-wide maximum time budget $T_{\max }$ (possibly automatically computed, see Subsection V-A). Once got access to the disk, each active application must consume all of its time budget or backlog within no more than $T_{\max }$ time units, otherwise it is unconditionally (over)charged for a $B_{i, \max }$ service and the next active application is selected. This additional mechanism prevents applications performing random IO from substantially decreasing the disk throughput. Hence it guarantees practical bandwidths and delays, which are inversely proportional to the aggregate throughput (Subsec. III-B), to applications performing mostly sequential IO. In contrast, applications performing random IO virtually receive no service guarantees.
According to (1) and (2), in addition to influencing the disk throughput, all these parameters directly or indirectly influence also guarantees. Hence, to set the desired trade-off between guarantee granularity and throughput boosting, the values of these parameters must be (iteratively) tuned by (iteratively) measuring the resulting throughput. BFQ also provides a simplified interface, which allows a user not interested in full control over all the parameters to avoid the resulting tuning complexity. This interface has been used in the experiments reported in Section V, and is described in detail in Subsection V-A.

Whatever interface is used, to evaluate both the (worst-case) throughput and the (worst-case) guarantees as a function of the parameters, and to tune the latter, it is necessary to measure the aggregate throughput against some (worst-case) benchmark request pattern. Such a pattern may be defined as a function of the expected one. For example, the following conservative worst-case pattern may be used to evaluate the expected minimum aggregate throughput for simultaneous sequential reads. After placing two files with size $S_{\min }$, equal to the (portions of the) files that will be interested by sequential accesses, at the maximum possible distance in $\left[P_{\text {first }}, P_{\text {last }}\right]$, where $P_{\text {last }}-P_{\text {first }}$ is the maximum span of the positions of the requests issued by the applications, a simultaneous sequential read of the two files may be performed. As confirmed also by our experiments, the resulting aggregate throughput provides a lower bound to the aggregate throughput for parallel file reads as well as Web server workloads. For random workloads, a request arrival pattern with the same locality as the expected one can be used to estimate the expected aggregate throughput. An example of the aggregate throughput achieved with a random workload is reported in Subsection V-F.

\section{RELATED WORK}

Existing algorithms for providing a predictable disk service can be broadly divided into three groups: 1) real-time disk schedulers [19], ; 2) proportional share or bandwidth reservation timestampbased disk schedulers (also known as fair-queueing schedulers); and 3) proportional share round robin disk schedulers. In addition, examples of frameworks for providing QoS guarantees are Cello [20], APEX [21], PRISM [22] and Argon [23]. It is worth mentioning also real-time operating systems such as the Dresden Real-Time Operating System (DROPS) [5] and RT-Mach [6], and Real-Time Database Systems (RTDBS), which are architectures for performing database operations with real-time constraints [24].

There is no relation between any of the scheduling problems highlighted in this paper, namely loss of throughput and/or guarantees due to deceptive idleness and/or delayed arrivals, and any characteristic of the above mentioned frameworks for QoS provisioning and RTDBSes (apart from which underlying scheduling algorithm(s) they rely on). Accordingly, after the following note about disk internal queueing, in the next subsections we focus only on each of the above listed classes of scheduling algorithms.

Disk internal queueing can be used only if disk idling is disabled, which ultimately causes loss of guarantees with any scheduler/framework for applications issuing synchronous requests. Consider, e.g., a proportional share scheduler, and suppose that the $i$-th application has an arbitrarily high weight. After each request $R_{i}^{j}$ of the application is completed, the disk starts serving its next internally queued request, or immediately asks for a new request, without waiting for $R_{i}^{j+1}$ to arrive. Hence another application is served, independently of whether $R_{i}^{j+1}$ would then 
happen to be the next request to serve. In the end, the guarantees assigned to the application may be easily violated.

\section{A. Real-time Schedulers}

Real-time schedulers [4], [19] are timestamp-based schedulers that associate a deadline to each request. They usually start from an Earliest Deadline First (EDF) [25] schedule, and reorder requests to reduce seek and rotational latency without violating deadlines.

Each time the next request to serve must be picked, RotationalPosition-Aware disk scheduling based on a Dynamic Active Subset (SATF-DAS) [5] iteratively constructs and serves a subset of the outstanding requests, called Dynamic Active Subset (DAS) and such that any throughput boosting algorithm can be used to order the requests in the DAS without violating service guarantees. Defined as slack of a request the difference between the deadline of the request and the earliest time by which the request can be served, Just-In-Time Slack Stealing (JIT) [6] is based on serving requests closer to the disk head instead of requests with lower deadlines but with large enough positive slack. SCAN-EDF [4] serves requests in EDF order, but if several requests have the same deadline, they are scheduled using a seek optimization algorithm (e.g., SCAN or C-LOOK). Finally, other proposals are Priority SCAN (PSCAN), Earliest Deadline SCAN and Feasible Deadline SCAN (FD-SCAN) [24], which are quite similar in principle to the above described ones.

With regard to throughput boosting, in both SATF-DAS and JIT there is no control either on the size of the DAS, or on the amount of available slack. In contrast, the authors of SCANEDF propose enlarging the request size and extending relative deadlines beyond the period to effectively trade response time and buffer requirements for throughput boosting.

Problems arise if applications issuing synchronous requests are scheduled with real-time bandwidth servers built on top of the above real-time schedulers. First, as all of these schedulers are work-conserving, throughput is likely to be very low. However, this problem can be easily solved by extending these schedulers, where possible, to perform disk idling as in BFQ, CFQ or AS. The second problem follows from the fact that deadlines may be missed, mainly because of the non-preemptability of the service of a request. The consequent delayed arrivals, plus the fact that in bandwidth servers the absolute deadlines of the requests are usually computed by summing their relative deadlines to their arrival times, may cause delayed requests to be unjustly assigned higher deadlines. As discussed in the introduction, this may cause the desired bandwidth distribution to be violated. Both problems clearly manifest themselves in our experimental results (Section V).

\section{B. Proportional Share Timestamp-based Schedulers}

YFQ [8] dispatches requests to the disk device in batches. In particular, before the next batch is served, all the requests in the current batch are dispatched. The requests to insert in each batch are chosen using WFQ [14], and may be ordered with the desired throughput boosting algorithm within the batch. The fact that the limited room in a batch is in general filled with requests issued by all the backlogged applications reduces the probability of inserting a high number of requests of the same application. This may reduce the number of close or sequential accesses with mainstream applications performing sequential IO. Moreover, guarantees may be violated for an application issuing synchronous requests: even if the application has a (much) higher weight than the others, no more than one request of the application will be served for each batch.

SFQ(D) and FSFQ(D) [12] allow a configurable number of outstanding requests $D$ to be dispatched, where, each time one of the outstanding requests completes, the next one is immediately dispatched. SFQ(D) selects requests according to a variant of SFQ that does not suffer from loss of fairness in presence of applications not consuming their fair share of the disk throughput. FSFQ(D) is a further refinement of SFQ(D) that tries to compensate the possible loss of service of an application due to the late arrival of its requests. Similarly to SFQ(D) and FSFQ(D), Hybrid [7] allows a configurable amount of outstanding requests to be chosen by an internal WF2Q+ scheduler, and to be reordered by the desired throughput boosting algorithm. To avoid starvation Hybrid periodically flushes all the outstanding requests. As in BFQ the internal scheduler works in the service domain. Finally, pClock [9] is based on a more general scheme: through arrival curves, application requirements are expressed in terms of throughput, latency and maximum burst size. Applications following their arrival curve are proven to never miss their deadlines.

Differently from BFQ and Hybrid, YFQ, SFQ(D), FSFQ(D) and pClock directly target proportional time allocation instead of sector allocation, and the accuracy of their disk throughput distribution depends on the accuracy in estimating request service times. The main problem is however that, as real-time schedulers, all the schedulers mentioned in this section timestamp requests as a function of their actual arrival times. Hence, independently of whether disk idling is performed, they may fail to distribute the bandwidth as desired in presence of synchronous requests. Our experimental results confirm this problem.

\section{Round Robin Schedulers}

CFQ is a proportional share disk scheduler that grants disk access to each application for a fixed time slice. Slices are scheduled according to a round robin policy. This time-based allocation, equal to the one adopted in the Argon framework, has the advantage of implicitly charging each application for the seek and rotational latencies it incurs. Unfortunately this scheme may suffer from unfairness problems also towards applications making the best possible use of the disk bandwidth. Even if the same time slice is assigned to two applications, they may get a different throughput each, as a function of the positions on the disk of their requests. BFQ owes to CFQ the idea of exclusively serving each application for a while, but provides strong guarantees on bandwidth distribution because the assigned budgets are measured in number of sectors.

Strong service distribution guarantees on a per-request basis are provided also by the adaptive Deficit Round Robin proposed in [10], as it measures the amount of service received by any application in terms of number of requests served. To achieve the desired trade-off between fairness and I/O efficiency the scheduler is also configurable in terms of maximum number $D$ of outstanding requests and maximum per-application number $G_{i}$ of requests dispatched in each round. Unfortunately, as any round robin scheduler, both this scheduler and CFQ are characterized by an $O(N)$ worst-case jitter in request completion time, where 
$N$ is the number of competing applications. In contrast, thanks to the accurate service distribution of the internal $\mathrm{B}-\mathrm{WF}^{2} \mathrm{Q}+$ scheduler, BFQ exhibits $O(1)$ jitter according to (2) with respect to the number of applications. A quantitative evaluation of the consequences of this different short term guarantees and of the above mentioned unfairness of CFQ is reported in Section V.

\section{EXPERIMENTAL RESULTS}

In this section we report the results of our single-disk experiments with BFQ, SCAN-EDF, YFQ, CFQ, C-LOOK, and AS, on a system running the Linux 2.6.21 kernel. We first provide implementation and configuration details in the next subsection. Then we describe the experimental setup and report the results of each set of experiments in the successive ones.

\section{A. Scheduler implementation and configuration}

If the simplified interface of BFQ is used, $T_{F I F O}$ is set to the default value used by the other schedulers in the system (typically $100 \mathrm{~ms}$ ), $T_{\max }$ is dynamically set/updated to $1 . \overline{3}$ times the average time needed to consume $B_{\max }$ sectors, $\forall i B_{i, \max }$ is set to $B_{\max }$, and either a throughput boosting level ranging from 0 to 1 , or just $B_{\max }$ are exported as the only configuration parameter. In the first case, the back-end of the interface will take care of setting $B_{\max }$ accordingly, from the minimum possible request size, to the number of sectors served in $200 \mathrm{~ms}$. The latter value is automatically computed/updated [16] and guarantees a high throughput, as shown in Subsection V-C. We used the simplified interface and set only the maximum budget $B_{\max }$ in our experiments.

As no code of SCAN-EDF and YFQ was available for the Linux kernel, we implemented a slightly extended version of each of them in the 2.6.21 Linux kernel [16]. In our implementation of SCAN-EDF, each application is associated with a dynamically configurable relative deadline, equal e.g., to the application's period. This relative deadline is assigned to each request issued by the application. The resulting algorithm can be seen as a simple real-time bandwidth server. Suppose that the $i$-th application issues requests of the same size $L_{i}$ back-to-back, and that a relative deadline equal to $P_{i}$ is assigned to all of its requests. The computed absolute deadlines of the requests will be the same as if the application was periodic with period $P_{i}$. Hence, in a fullloaded system, the application should be guaranteed a fraction of the bandwidth equal to $L_{i} / P_{i}$. Finally, to deal with the deceptive idleness, after the completion of the last request of an application, both implementations keep the disk idle until either a new request of the just served application arrives, or a configurable $T_{\text {wait }}$ time interval elapses.

To trade response time for throughput boosting the authors of SCAN-EDF suggest both to change the request size and the coarsen deadline granularity. Unfortunately, the first strategy cannot be used in the Linux kernel, as the request size is not controlled by the disk schedulers. In contrast, our implementation of SCAN-EDF allows a system-wide granularity parameter $\Delta$ to be set. Given a request with absolute deadline $d$, and the smallest $n$ such that $n \cdot \Delta \geq d$, the request is scheduled as if its deadline was $n \cdot \Delta$. With regard to YFQ, the batch size, measured in number of requests, is configured through the $B T_{\text {size }}$ parameter. Batch overlapping, one of the enhancements proposed by the authors of YFQ to increase disk throughput, is performed as well (see the code [16]). All the requests with the same deadline and all the requests in a batch are served in C-LOOK order.

\section{B. Experiments}

The experiments were aimed at measuring the aggregate throughput, long-term bandwidth distribution and (short-term) per-request completion time guaranteed by the six schedulers with the following applications/workloads: simultaneous sequential reads, Web server (emulated), DBMS (emulated) and mixed video-streaming/file reads. All these applications only issue synchronous requests on a Linux system. In addition, as discussed at the end of Subsection V-C the same results in terms of aggregate throughput would be achieved with all these applications, apart from DBMS, in case they would issue asynchronous requests. Hence, only in the DBMS case we also showed the different performance of the schedulers with an asynchronous workload. Due to space limitations, only a synthesis of the results is reported here. The complete results and all the programs used to generate them can be found in [16].

We ran the experiments on a PC equipped with a $1 \mathrm{GHz}$ AMD Athlon processor, $768 \mathrm{MB}$ RAM, and a 30 GB IBM-DTLA307030 ATA IDE hard drive (roughly $36 \mathrm{MB} / \mathrm{sec}$ peak bandwidth in the outer zones, $\sim 35 \%$ lower throughput in the inner zones), accessed in UDMA mode. Using this low performance disk device helped us guarantee that the disk was the only bottleneck. Sectors were 512 bytes long (ext 2 file-system). The disk was partitioned into 30 consecutive slices of equal size, the first slice covering the outer part of the disk, the last one covering the inner part. All the programs were run from an auxiliary disk. For each type of experiment and set of values of the parameters, the same experiment was repeated 20 times (the buffer cache was flushed before each experiment). The minimum, maximum, and mean value, together with its associated $95 \%$ confidence interval were computed for each output quantity. In what follows any mean value $v$ is reported in the form $v \pm s$, where $s$ is the semi-width of the $95 \%$ confidence interval for $v$.

\section{Aggregate throughput}

The first set of experiments was aimed at estimating the worstcase aggregate throughput guaranteed by each scheduler in case of simultaneous sequential reads. As can be seen in the next subsections, according to our experiments these results hold also for a Web server workload. Under BFQ and YFQ, all applications were assigned the same weight, whereas they were assigned the same priority under CFQ (which allows applications to be assigned different priorities). Under SCAN-EDF all the requests were assigned the same deadline, equal to $20 \mathrm{~ms}$. The whole set of different experiments was given by the combinations of the following 5 values: scheduler in $\mathrm{BFQ}$, SCAN-EDF, YFQ, CFQ, C-LOOK, AS $\}$; cardinality of the set of distinct files to read in $\{2,3,4,5\}$ (for each set, the files were placed in slices at the maximum possible distance from each other, with each file in a distinct slice); value of the scheduler configuration parameter: maximum budget $B_{\max }$ in $\{512,1024,2048,4096,8192,16384\}$ sectors for BFQ, batch size $B T_{\text {size }}$ in $\{4,8,16\}$ requests for YFQ, deadline granularity $\Delta$ in $\{20,40,80,160,320\}$ ms for SCAN$\mathrm{EDF}$, and time slice $T_{\text {slice }}$ equal to $100 \mathrm{~ms}$ (the default value) for CFQ; $T_{\text {wait }}$ in $\{0,4\} \mathrm{ms}$ for SCAN-EDF and YFQ, and $T_{\text {wait }}=4 \mathrm{~ms}$ for BFQ (CFQ automatically sets/changes $T_{\text {wait }}$ ); 


\begin{tabular}{c||c|c}
\hline Scheduler & $\begin{array}{c}\text { Mean Agg. Thr } \\
{[\mathrm{MB} / \mathrm{s}]}\end{array}$ & $\begin{array}{c}\text { Value of } B_{\max } \\
T_{\text {slice }}, \Delta, B T_{\text {size }}\end{array}$ \\
\hline \hline BFQ & $22.46 \pm 0.81$ & $16384 \mathrm{sect}$ \\
\hline SCAN-EDF & & \\
$T_{\text {wait }}=0 \mathrm{~ms}$ & $21.18 \pm 0.47$ & $640 \mathrm{~ms}$ \\
$T_{\text {wait }}=4 \mathrm{~ms}$ & $23.39 \pm 0.51$ & \\
\hline YFQ & & \\
$T_{\text {wait }}=0 \mathrm{~ms}$ & $10.64 \pm 0.25$ & $16 \mathrm{reqs}$ \\
$T_{\text {wait }}=4 \mathrm{~ms}$ & $10.80 \pm 0.20$ & \\
\hline CFQ & $16.91 \pm 1.30$ & $100 \mathrm{~ms}$ \\
\hline C-LOOK & $20.59 \pm 0.76$ & \\
\hline AS & $32.97 \pm 1.89$ & \\
\hline
\end{tabular}

TABLE II

AGGREGATE THROUGHPUT FOR TWO SIMULTANEOUS READS.

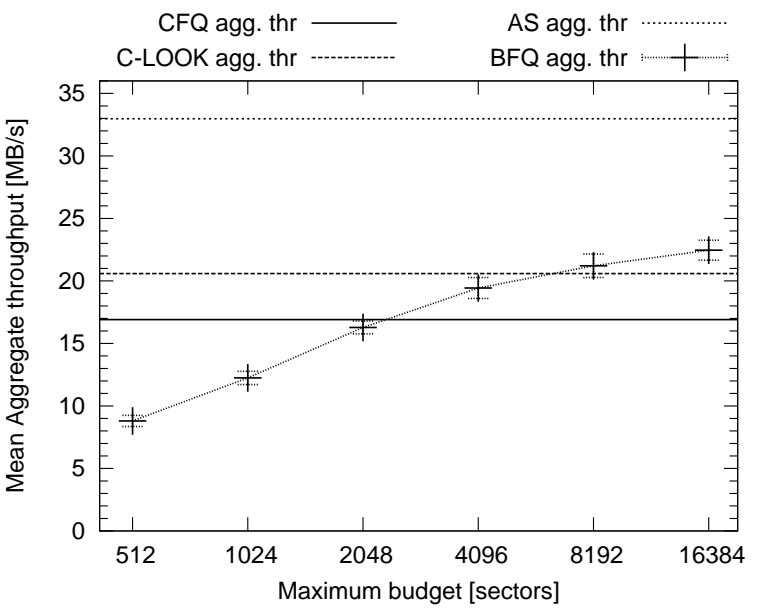

Fig. 5. Mean aggregate throughput, and associated $95 \%$ conf. interval, achieved by BFQ (as a function of $B_{\max }$ ), CFQ, C-LOOK and AS in case of simultaneous reads of two, $128 \mathrm{MB}$ long, files.

CFQ. Finally, it can be seen that the aggregate throughput with AS is close to the disk peak rate, which means that exclusively serving each application for a certain amount of time-as AS, CFQ and BFQ do-leads in practice to the highest possible throughput if applications issue sequential requests. This also implies that SCAN-EDF and YFQ certainly would not achieve a higher throughput if these applications issued asynchronous requests.

\section{Simultaneous sequential reads}

The second feature we evaluated is the accuracy of the schedulers in providing the desired bandwidth fraction to applications performing sequential reads. For brevity, for BFQ we only report the results for $B_{\max }=4096$ sectors. Similarly, for SCAN-EDF, we consider only $\Delta=80 \mathrm{~ms}$ because for this value SCAN-EDF achieves an aggregate throughput close to BFQ. Finally, in our experiments the batch size had no influence on the guarantees provided by YFQ. We report the results for $B T_{\text {size }}=4$ requests.

We first considered the case where all the applications are allocated the same fraction of the disk bandwidth. In particular, during the same experiments used to evaluate the aggregate throughput, we measured the throughput of each (file read) application. For each scheduler, the highest deviation from the ideal distribution occurred for $128 \mathrm{MB}$ long files. Moreover, we observed that the $95 \%$ confidence interval for the mean throughput of the $i$-th application in case of 3 and 4 files was always smaller (or greater) than that obtained in case in case of 5 (or 2) files. This inclusion property held also in case of asymmetric allocation (see below). Hence, for brevity, we report only the results for two and five, $128 \mathrm{MB}$ long, files. Finally, we consider only $T_{\text {wait }}=4$ ms for SCAN-EDF and YFQ in Table III.

BFQ, YFQ and C-LOOK exhibit the most accurate bandwidth distribution (C-LOOK basically performs a round robin among the batch of requests issued by the read-ahead mechanism). Unfortunately, as previously seen, YFQ has a low throughput. SCANEDF is less accurate for 2 files, but it provides a higher throughput than YFQ. Consistently with the arguments in Subsection IV$\mathrm{B}$, CFQ fails to fairly distribute the throughput because of the varying sector transfer speed. Finally, as expected AS has the most asymmetric bandwidth distribution. Moreover the high width of the confidence interval for AS is a consequence of the fact that sometimes the waiting timer may expire before the next $\mathrm{CFQ}, \mathrm{C}-\mathrm{LOOK}$ and $\mathrm{AS}$ are reported as a reference too. For $B_{\max }=4096$ sectors BFQ guarantees a higher throughput than 


\begin{tabular}{|c|c|c|c|c|c|}
\hline $\begin{array}{c}\text { Throughput } \\
\text { (2 files) }\end{array}$ & $\begin{array}{c}9.95 \\
\pm 0.43\end{array}$ & & & & $\begin{array}{c}9.81 \\
\pm 0.47\end{array}$ \\
\hline $\begin{array}{l}\text { Throughput } \\
\text { (5 files) }\end{array}$ & $\begin{array}{c}4.29 \\
\pm 0.10\end{array}$ & $\begin{array}{c}4.30 \\
\pm 0.09\end{array}$ & $\begin{array}{c}4.30 \\
\pm 0.07\end{array}$ & $\begin{array}{c}4.29 \\
\pm 0.10\end{array}$ & $\begin{array}{c}4.31 \\
\pm 0.09\end{array}$ \\
\hline \multicolumn{6}{|c|}{ BFQ $\left(B_{\max }=4096\right.$ sectors $)$} \\
\hline $\begin{array}{c}\text { Throughput } \\
\text { (2 files) }\end{array}$ & $\begin{array}{l}10.72 \\
\pm 0.22\end{array}$ & & & & $\begin{array}{c}9.62 \\
\pm 0.19\end{array}$ \\
\hline $\begin{array}{l}\text { Throughput } \\
\text { (5 files) }\end{array}$ & $\begin{array}{c}2.17 \\
\pm 0.02\end{array}$ & $\begin{array}{c}2.17 \\
\pm 0.02 \\
\end{array}$ & $\begin{array}{c}2.17 \\
\pm 0.02 \\
\end{array}$ & $\begin{array}{c}2.17 \\
\pm 0.02\end{array}$ & $\begin{array}{c}2.19 \\
\pm 0.03\end{array}$ \\
\hline \multicolumn{6}{|c|}{ SCAN-EDF $\left(\Delta=80 \mathrm{~ms}, T_{\text {wait }}=4 \mathrm{~ms}\right)$} \\
\hline $\begin{array}{c}\text { Throughput } \\
\text { (2 files) }\end{array}$ & $\begin{array}{c}5.44 \\
\pm 0.10\end{array}$ & & & & $\begin{array}{c}5.44 \\
\pm 0.10\end{array}$ \\
\hline $\begin{array}{l}\text { Throughput } \\
\text { (5 files) }\end{array}$ & $\begin{array}{c}1.39 \\
\pm 0.02\end{array}$ & $\begin{array}{c}1.39 \\
\pm 0.02\end{array}$ & $\begin{array}{c}1.39 \\
\pm 0.02\end{array}$ & $\begin{array}{c}1.39 \\
\pm 0.02\end{array}$ & $\begin{array}{c}1.39 \\
\pm 0.03\end{array}$ \\
\hline \multicolumn{6}{|c|}{ YFQ $\left(B T_{\text {size }}=4\right.$ requests, $\left.T_{\text {wait }}=4 \mathrm{~ms}\right)$} \\
\hline $\begin{array}{l}\text { Throughput } \\
\text { (2 files) }\end{array}$ & $\begin{array}{l}11.92 \\
\pm 0.44\end{array}$ & & & & $\begin{array}{c}8.61 \\
\pm 0.67\end{array}$ \\
\hline $\begin{array}{l}\text { Throughput } \\
\text { (5 files) }\end{array}$ & $\begin{array}{c}5.24 \\
\pm 0.14 \\
\end{array}$ & $\begin{array}{c}4.91 \\
\pm 0.15 \\
\end{array}$ & $\begin{array}{c}4.66 \\
\pm 0.11 \\
\end{array}$ & $\begin{array}{c}4.37 \\
\pm 0.10 \\
\end{array}$ & $\begin{array}{c}4.01 \\
\pm 0.17 \\
\end{array}$ \\
\hline \multicolumn{6}{|c|}{$\mathbf{C F Q}\left(T_{\text {slice }}=100 \mathrm{~ms}\right)$} \\
\hline $\begin{array}{l}\text { Throughput } \\
\text { (2 files) }\end{array}$ & $\begin{array}{l}11.52 \\
\pm 1.78\end{array}$ & & & & $\begin{array}{l}10.57 \\
\pm 0.55\end{array}$ \\
\hline $\begin{array}{l}\text { Throughput } \\
\text { (5 files) }\end{array}$ & $\begin{array}{c}5.56 \\
\pm 0.67\end{array}$ & $\begin{array}{c}5.14 \\
\pm 0.41\end{array}$ & $\begin{array}{c}5.05 \\
\pm 0.50\end{array}$ & $\begin{array}{c}4.95 \\
\pm 0.35\end{array}$ & $\begin{array}{c}4.83 \\
\pm 0.09\end{array}$ \\
\hline \multicolumn{6}{|c|}{ C-LOOK } \\
\hline $\begin{array}{c}\text { Throughput } \\
\text { (2 files) }\end{array}$ & $\begin{array}{c}32.41 \\
\pm 13.39\end{array}$ & & & & $\begin{array}{l}17.78 \\
\pm 1.10\end{array}$ \\
\hline $\begin{array}{l}\text { Throughput } \\
\text { (5 files) }\end{array}$ & $\begin{array}{c}32.83 \\
\pm 11.95\end{array}$ & $\begin{array}{c}31.72 \\
\pm 1.37\end{array}$ & $\begin{array}{l}29.80 \\
\pm 0.50\end{array}$ & $\begin{array}{c}12.56 \\
\pm 10.46\end{array}$ & $\begin{array}{l}18.29 \\
\pm 0.43\end{array}$ \\
\hline
\end{tabular}

AS

TABLE III

SiMULTANEOUS SEQUENTIAL READS

synchronous request arrives. In that case also AS switches to the service of another application.

It is important to observe that the accurate throughput distribution of YFQ and SCAN-EDF is mostly related to the symmetry of the bandwidth allocation. To measure the accuracy of the schedulers in distributing the disk bandwidth in case of asymmetric allocations, under BFQ and YFQ we assigned different weights to the applications. We run two sets of experiments, using, respectively, the weights 1 and 2 , and the weights 1,2 and 10 (there is no constraint on the values of the weights in our implementations of BFQ and YFQ). Moreover, assuming that the $j$-th application is the one with maximum weight, and denoted as $S_{j}$ the size of the file read by the $j$-th application, the file read by the $i$-th application had a length $\frac{\phi_{i}}{\phi_{j}} S_{j}$. To try to allocate the same bandwidth as with BFQ and YFQ, under SCAN-EDF we assigned to each request of an application a relative deadline inversely proportional to the weight assigned to the application with the other two schedulers. Finally, this type of experiments was not run for CFQ, C-LOOK and AS, which do not provide differentiated bandwidth allocations.

To show the worst-case, yet not distorted by external factors, performance of the schedulers, for the scenarios where the maximum weight was, respectively, 2 and 10 we report the results of only the experiments where the maximum file sizes were, respectively, $256 \mathrm{MB}$ and $1 \mathrm{~GB}$. Finally, since the above defined

\begin{tabular}{c||c|c|c|c|c}
\hline Weight & 2 & 2 & 2 & 1 & 1 \\
\hline $\begin{array}{c}\text { Throughput } \\
\text { (2 files) }\end{array}$ & $\begin{array}{c}14.62 \\
\pm 0.60\end{array}$ & & & & $\begin{array}{c}7.31 \\
\pm 0.32\end{array}$ \\
\hline $\begin{array}{c}\text { Throughput } \\
\text { (5 files) }\end{array}$ & $\begin{array}{c}5.60 \\
\pm 0.08\end{array}$ & $\begin{array}{c}5.60 \\
\pm 0.08\end{array}$ & $\begin{array}{c}5.60 \\
\pm 0.08\end{array}$ & $\begin{array}{c}2.81 \\
\pm 0.04\end{array}$ & $\begin{array}{c}2.81 \\
\pm 0.04\end{array}$ \\
\hline \hline Weight & 10 & 2 & 2 & 1 & 1 \\
\hline $\begin{array}{c}\text { Throughput } \\
\text { (2 files) }\end{array}$ & 26.81 & & & & 2.77 \\
\pm 0.60 & & & & \pm 0.16 \\
\hline $\begin{array}{c}\text { Throughput } \\
\text { (5 files) }\end{array}$ & 16.06 & 3.25 & 3.25 & 1.64 & 1.65 \\
\pm 0.28 & \pm 0.07 & \pm 0.07 & \pm 0.04 & \pm 0.04 \\
\hline
\end{tabular}

TABLE IV

BFQ WITH ASYMMETRIC WEIGHTS.

inclusion property holds also in case of asymmetric allocation, for brevity we report the results with BFQ only for the 2 and 5 files scenarios in Table IV.

With regard to SCAN-EDF and YFQ, in accordance with what is said in Subsections IV-A and IV-B, both failed to guarantee the desired bandwidth distribution in all the experiments. For example, in case of 2 applications with weights 10 and 1 , the throughputs were $\{26.58 \pm 0.50,6.92 \pm 0.20\} \mathrm{MB} / \mathrm{s}$ for SCAN$\mathrm{EDF}$, and $\{22.70 \pm 0.12,5.41 \pm 0.12\} \mathrm{MB} / \mathrm{s}$ for YFQ (the skewness is even attenuated by the fact that the application with a higher weight has to read a longer file, and hence it gets exclusive access to the disk after the other one finished).

\section{E. Web server}

In this set of experiments we estimated the per-request completion time guaranteed by the schedulers against the following Web server-like workload: 100 processes (all with the same weight/priority) continuously read files one after the other. Each of the files to read may have been, with probability 0.9, a small $16 \mathrm{kB}(\mathrm{html})$ file at a random position in the first half of the disk, or, with probability 0.1 , a large file with random size in $[1,30] \mathrm{MB}$ at a random position in the second half of the disk. Every 10 files, each process appended a random amount of bytes, from 1 to 16 $\mathrm{kB}$, to a common log file. Such a scenario allows the performance of the schedulers to be measured for a mainstream application and, in general, in presence of a mix of both sequential (large files) and a random (small files) requests. Especially, for each run, lasting for about one hour, we measured the completion time of each small file (latency), the (average) bandwidth at which large files were read and the average aggregate throughput (measured every second). In this respect, small and large file reads were performed in separated parts of the disk to generate an asymmetric workload, which is more prone to higher latencies and/or lower bandwidths.

As can be seen from Table V-excluding for a moment SCANEDF and YFQ-BFQ, CFQ and AS stand, respectively at the beginning, (about) the middle and the end of the low latency versus high aggregate throughput scale. Also C-LOOK achieves similar performance as AS, because a high number of competing requests scattered over all the entire disk are present at all times. In contrast YFQ has very low latencies and throughput because, as the probability of a small file read is 9 times higher than the one of a large file read, each batch is likely to contain a high percentage of requests pertaining to small files. Finally, although achieving a lower aggregate throughput than BFQ, SCAN-EDF 


\begin{tabular}{c||c|c|c}
\hline Scheduler & $\begin{array}{c}\text { Compl. time } \\
\text { small files } \\
\text { [sec] }\end{array}$ & $\begin{array}{c}\text { Bandw } \\
\text { large files } \\
\text { [sec] }\end{array}$ & $\begin{array}{c}\text { Mean aggr. } \\
\text { throughput } \\
{[\mathrm{MB} / \mathrm{s}]}\end{array}$ \\
\hline \hline BFQ & $1.74 \pm 0.11$ & $2.26 \pm 2.34$ & $17.13 \pm 0.65$ \\
\hline SCAN-EDF & $7.68 \pm 0.20$ & $3.29 \pm 3.10$ & $8.71 \pm 0.09$ \\
\hline YFQ & $0.40 \pm 0.01$ & $1.36 \pm 1.71$ & $6.87 \pm 0.09$ \\
\hline CFQ & $3.86 \pm 0.17$ & $2.97 \pm 2.47$ & $18.20 \pm 0.79$ \\
\hline C-LOOK & $7.78 \pm 0.27$ & $9.65 \pm 3.93$ & $19.47 \pm 0.66$ \\
\hline AS & $7.69 \pm 0.27$ & $16.61 \pm 4.00$ & $20.60 \pm 1.22$ \\
\hline
\end{tabular}

TABLE V

WEB SERVER (RANDOM/SEQUENTIAL READS).

guarantees higher bandwidths to the large files, by sacrificing the latency of the small ones. It is worth noting that the observed mean latency of BFQ is 2.22 times lower than CFQ and at least 4.41 times lower than all the other schedulers. On the contrary, with any scheduler, the high width of the confidence interval for the mean bandwidth of the large files is a consequence of the quite random nature of the workload.

To check whether the guarantee (2) complies with the observed latencies for small files, we can set $t_{1}$ to the arrival time of the first request of a generic small file, and assume that $R_{i}^{j}$ is the last request for that file, which implies $Q_{i}\left(t_{1}^{-}\right)+A\left(t_{1}, a_{i}^{j}\right)=$ $16 k B$ (as explained in Subsection III-B $T_{F I F O}$ can be neglected with synchronous requests). Moreover, $\phi_{i}=\frac{1}{100}, L_{\max }=256$ sectors in the Linux kernel (by default), and in the experiments we found that if the $i$-th application is one of the processes reading small files, then $L_{i, \min } \equiv \min _{j} L_{i}^{j}=16$ sectors and a budget oscillating from 8 to 256 sectors is assigned to the application. Hence, setting $B_{i, \max }=256$ sectors and using the mean throughput $T_{a g g}=17.13$, the resulting guaranteed latency is $\frac{100 *(16+(128-8)+128)+(2048+2 * 128)}{17.13 * 1024}=1.64$ seconds. This value is $\sim 6 \%$ lower than the observed mean latency, which is most certainly due to the fact that when small files are read the throughput falls down to a lower value than the mean used in the formula.

\section{F. DBMS}

This set of experiments was aimed at estimating the request completion time and the aggregate throughput achieved by the schedulers against a DBMS-like workload: 100 processes (all with the same weight/priority) concurrently issue direct (non cacheable) $4 \mathrm{~KB}$ read requests at random positions in a common $5 \mathrm{~GB}$ file. We have used this setup to evaluate also the performance of the schedulers with asynchronous requests, and, to this purposes, we have run 10 variants of the experiment, with each variant characterized by a different number of per-process outstanding requests, ranging from 1 (synchronous requests) to 10. According to our results, for each scheduler both the request completion time and the aggregate throughput monotonically grew with the number of outstanding requests. Hence, for brevity we report in Table VI the mean request completion time and aggregate throughput only for 1 and 10 outstanding requests.

With random requests, AS does not perform disk idling at all, hence it achieves the same results as pure C-LOOK. Especially, thanks to their global position-based request ordering, C-LOOK and AS achieve the lowest request completion time and hence the highest disk throughput. Being its service scheme closer to

\begin{tabular}{c||c|c|c}
\hline Scheduler & $\begin{array}{c}\text { Num. of } \\
\text { outstand. } \\
\text { requests }\end{array}$ & $\begin{array}{c}\text { Completion } \\
\text { time } \\
{[\mathrm{sec}]}\end{array}$ & $\begin{array}{c}\text { Mean aggr. } \\
\text { throughput } \\
{[\mathrm{MB} / \mathrm{s}]}\end{array}$ \\
\hline \hline BFQ & 1 & $0.92 \pm 0.12$ & $0.44 \pm 0.00$ \\
& 10 & $7.93 \pm 0.29$ & $0.49 \pm 0.00$ \\
\hline SCAN-EDF & 1 & $0.93 \pm 0.00$ & $0.44 \pm 0.00$ \\
& 10 & $6.49 \pm 0.85$ & $0.60 \pm 0.00$ \\
\hline YFQ & 1 & $0.69 \pm 0.01$ & $0.59 \pm 0.00$ \\
& 10 & $6.59 \pm 0.06$ & $0.59 \pm 0.00$ \\
\hline CFQ & 1 & $0.91 \pm 0.09$ & $0.45 \pm 0.00$ \\
& 10 & $8.01 \pm 0.30$ & $0.49 \pm 0.00$ \\
\hline C-LOOK/ & 1 & $0.67 \pm 0.01$ & $0.62 \pm 0.00$ \\
AS & 10 & $5.52 \pm 0.12$ & $0.71 \pm 0.00$ \\
\hline
\end{tabular}

TABLE VI

DBMS (RANDOM READS)

C-LOOK/AS than the one of BFQ, SCAN-EDF and CFQ, YFQ outperforms the latter in case of synchronous requests. It has instead the same performance as SCAN-EDF with 10 outstanding requests. Finally, because of their slice-by-slice/budget-by-budget service scheme both CFQ and BFQ exhibit a $\sim 1.4$ times higher request completion time/lower throughput than C-LOOK/AS. It is however worth noting that only $\sim 2 \%$ of the disk rate is achieved by the latter, which is typically unbearable in a realistic DBMS. This result complies with the fact that, if the disk requests enjoy some locality, caches are usually tuned so as to reduce disk access and achieve feasible response times (of course multiple disks are typically used as well).

\section{G. Video streaming}

The last set of experiments was aimed at measuring the ability of the schedulers to support a very time-sensitive application. To this purpose, we set up a VLC streaming server [26], provided with 30 movies to stream to remote clients. Each movie was stored in a distinct disk slice, and the streaming thread of the server that read it was considered as a distinct application by the disk schedulers (all the threads were assigned the same weight/priority). To evaluate the performance of the schedulers, a packet sent by the streaming thread was considered lost if delayed by more than one second with respect to its due transmission time (i.e., we assumed a 1 second playback buffer on the client side).

Every 15 seconds the streaming of a new movie was started. Each experiment ended either if 15 seconds elapsed from the start of the streaming of the last available movie, or if the packet loss rate reached the $1 \%$ threshold (this value, as well as the 1 second threshold for the delay, has been experimentally chosen to trade off between achieving a very high video quality, and allowing the system to stream a high enough number of simultaneous films to clearly show the different performance of the six schedulers). To mimic the mixed workload of a general purpose storage system and to increase to workload so that the disk was the only bottleneck, during each experiment we also run 5 ON/OFF file readers, each reading a portion of random length in $[64,512] \mathrm{MB}$ of a file, and then sleeping for a random time interval in $[1,200]$ $\mathrm{ms}$ before starting to read a new portion of the same file (each file was stored in a different disk slice). $\Delta, B T_{\text {size }}$ and $T_{\text {slice }}$ for SCAN-EDF, YFQ and CFQ were set to $20 \mathrm{~ms}, 4$ requests and $20 \mathrm{~ms}$, as these are the maximum values for which these schedulers achieve the highest number of simultaneous streams. 


\begin{tabular}{c|c||c|c}
\hline Scheduler & Param. & $\begin{array}{c}\text { Mean Num. } \\
\text { of Movies }\end{array}$ & $\begin{array}{c}\text { Mean Agg. } \\
\text { Thr. }[\mathrm{MB} / \mathbf{s}]\end{array}$ \\
\hline \hline & $4096 \mathrm{sect}$ & $24.00 \pm 0.00$ & $7.56 \pm 0.87$ \\
BFQ & $8192 \mathrm{sect}$ & $23.95 \pm 0.42$ & $8.15 \pm 1.08$ \\
& $16384 \mathrm{sect}$ & $18.70 \pm 9.45$ & $12.78 \pm 5.64$ \\
\hline SCAN-EDF & $20 \mathrm{~ms}$ & $12.00 \pm 0.00$ & $8.93 \pm 0.22$ \\
\hline YFQ & $4 \mathrm{reqs}$ & $19.00 \pm 0.00$ & $5.85 \pm 0.55$ \\
\hline CFQ & $20 \mathrm{~ms}$ & $14.35 \pm 1.40$ & $12.59 \pm 2.12$ \\
\hline C-LOOK & & $1.8 \pm 1.16$ & $22.66 \pm 0.96$ \\
\hline AS & & $1.1 \pm 1.04$ & $28.39 \pm 5.36$ \\
\hline \multicolumn{2}{|c|}{}
\end{tabular}

TABLE VII

VIDEO STREAMING DISTURBED BY SEQUENTIAL READS.

Table VII shows the mean number of movies and the mean aggregate throughput achieved by the schedulers during the last five seconds before the end of each experiment.

As can be seen, with $B_{\max }=4096$ sectors BFQ guarantees a stable and higher number of simultaneous streams than all the other five schedulers. Interestingly, with BFQ also the aggregate throughput is comparable/higher than SCAN-EDF/YFQ. Most certainly this is a consequence of the low $\Delta$ with SCAN-EDF.

Finally, to check whether the worst-case delay $d_{i, \max }$ guaranteed by BFQ to the periodic soft real-time application represented by any of the concurrent VLC streams complies with the observed maximum delay, from Table VII we can consider that, just before the end of most experiments, in case of $B_{\max }=4096$ sectors, $24+5$ applications with equal weights are competing for the disk, and the mean throughput is $7.56 \mathrm{MB} / \mathrm{s}$. As in the Web server experiments we have that $L_{\max }=256$ sectors, $L_{i, \min } \equiv$ $\min _{j} L_{i}^{j}=16$ sectors and a budget never higher than 256 sectors is assigned to the generic $i$-th streaming thread of the video server. Hence, according to $(3), d_{i, \max } \leq \frac{29 *(128-8)+(2048+128)}{7.56 * 1024}$ $=0.73$ seconds. This value complies with the above mentioned 1 second threshold for considering a packet as late, assuming that a reasonable additional worst-case delay of $\sim 0.27$ seconds is added by the rest of the system (probably mostly due to the execution of the 29 threads on the CPU).

\section{CONCLUSIONS}

In this paper we dealt with the problem of providing service guarantees while simultaneously achieving a high disk throughput in presence of synchronous requests. This type of requests may cause work-conserving scheduler to fail to provide a high throughput, and timestamp-based schedulers to fail to enforce guarantees.

In this respect, we proposed BFQ, a new disk scheduler that combines disk idling and timestamp back-shifting to achieve a high throughput and preserve guarantees also in presence of synchronous requests.

\section{REFERENCES}

[1] S. Iyer and P. Druschel, "Anticipatory scheduling: A disk scheduling framework to overcome deceptive idleness in synchronous I/O," in 18th ACM Symposium on Operating Systems Principles, Oct. 2001.

[2] G. Lawton, "Powering down the computing infrastructure," Computer, vol. 40, no. 2, pp. 16-19, 2007.

[3] B. L. Worthington, G. R. Ganger, and Y. N. Patt, "Scheduling algorithms for modern disk drives," in SIGMETRICS '94: Proceedings of the 1994 ACM SIGMETRICS conference on Measurement and modeling of computer systems. New York, NY, USA: ACM, 1994, pp. 241-251.
[4] A. L. N. Reddy and J. Wyllie, "Disk scheduling in a multimedia I/O system," in MULTIMEDIA '93: Proceedings of the first ACM international conference on Multimedia. New York, NY, USA: ACM, 1993, pp. 225-233.

[5] L. Reuther and M. Pohlack, "Rotational-position-aware real-time disk scheduling using a dynamic active subset (DAS)," in RTSS '03: Proceedings of the 24th IEEE International Real-Time Systems Symposium. Washington, DC, USA: IEEE Computer Society, 2003, p. 374.

[6] A. Molano, K. Juvva, and R. Rajkumar, "Real-time filesystems. guaranteeing timing constraints for disk accesses in rt-mach," Real-Time Systems Symposium, 1997. Proceedings., The 18th IEEE, pp. 155-165, 2-5 Dec 1997

[7] L. Rizzo and P. Valente, "Hybrid: achieving deterministic fairness and high throughput in disk scheduling," in Proceedings of CCCT'04, 2004.

[8] J. Bruno, J. Brustoloni, E. Gabber, B. Ozden, and A. Silberschatz, "Disk scheduling with quality of service guarantees," in ICMCS '99: Proceedings of the IEEE International Conference on Multimedia Computing and Systems Volume II-Volume 2. Washington, DC, USA: IEEE Computer Society, 1999, p. 400.

[9] A. Gulati, A. Merchant, and P. J. Varman, "pclock: an arrival curve based approach for qos guarantees in shared storage systems," SIGMETRICS Perform. Eval. Rev., vol. 35, no. 1, pp. 13-24, 2007.

[10] A. Gulati, A. Merchant, M. Uysal, and P. J. Varman, "Efficient and adaptive proportional share i/o scheduling," Hewlett-Packard, Tech. Rep., November 2007. [Online]. Available: http://www.hpl.hp.com/techreports/2007/HPL-2007-186.pdf

[11] [Online]. Available: http://mirror.linux.org.au/pub/linux.conf.au/ 2007/video/talks/123.pdf

[12] W. Jin, J. S. Chase, and J. Kaur, "Interposed proportional sharing for a storage service utility," in SIGMETRICS '04/Performance '04: Proceedings of the joint international conference on Measurement and modeling of computer systems. New York, NY, USA: ACM, 2004, pp. 37-48.

[13] [Online]. Available: http://google-opensource.blogspot.com/ 2008/08/linux-disk-scheduler-benchmarking.html

[14] J. C. R. Bennett and H. Zhang, "Hierarchical packet fair queueing algorithms," IEEE/ACM Transactions on Networking, vol. 5, no. 5, pp. 675-689, 1997.

[15] D. Stephens, J. Bennett, and H. Zhang, "Implementing scheduling algorithms in high-speed networks," Selected Areas in Communications, IEEE Journal on, vol. 17, no. 6, pp. 1145-1158, Jun 1999.

[16] [Online]. Available: http://algo.ing.unimo.it/people/paolo/disk_sched

[17] D. Stiliadis and A. Varma, "A general methodology for designing efficient traffic scheduling and shaping algorithms," INFOCOM '97. Sixteenth Annual Joint Conference of the IEEE Computer and Communications Societies. Proceedings IEEE, vol. 1, pp. 326-335, 1997.

[18] P. Valente, "Extending $\mathrm{WF}^{2} \mathrm{Q}+$ to support a dynamic traffic mix," Advanced Architectures and Algorithms for Internet Delivery and Applications, 2005. AAA-IDEA 2005. First International Workshop on, pp. 26-33, 15-15 June 2005.

[19] S. Daigle and J. Strosnider, "Disk scheduling for multimedia data streams," 1994.

[20] P. J. Shenoy and H. M. Vin, "Cello: a disk scheduling framework for next generation operating systems," SIGMETRICS Perform. Eval. Rev., vol. 26, no. 1, pp. 44-55, 1998.

[21] T. P. K. Lund, V. Goebel, "APEX: adaptive disk scheduling framework with QoS support," Multimedia Systems, vol. 11, no. 1, pp. 45-59, 2005.

[22] A. L. N. Reddy, J. Wyllie, and K. B. R. Wijayaratne, "Disk scheduling in a multimedia I/O system," ACM Trans. Multimedia Comput. Commun. Appl., vol. 1, no. 1, pp. 37-59, 2005.

[23] M. Wachs, M. Abd-El-Malek, E. Thereska, and G. R. Ganger, "Argon: Performance insulation for shared storage servers," in In Proceedings of the 5th USENIX Conference on File and Storage Technologies. USENIX Association, 2007.

[24] B. Kao and H. Garcia-Molina, "An overview of real-time database systems," 1995.

[25] C. L. Liu and J. W. Layland, "Scheduling algorithms for multiprogramming in a hard-real-time environment," $J . A C M$, vol. 20, no. 1, pp. 46-61, 1973.

[26] [Online]. Available: http://www.videolan.org/vlc/

\section{APPENDIX \\ PROOFS}

In this Appendix we provide the proofs of Theorems 1 and 2. Before proceeding, we introduce some additional notations and 
prove a lemma used in both proofs.

Since it is often used, for brevity we call total service property the fact that, at any time $t$, the total amount of service $W(t)$ provided by the real and the ideal system during $[0, t]$ is the same. Moreover, since we focus only on what happens inside $\mathrm{B}-\mathrm{WF}^{2} \mathrm{Q}+$, for simplicity from now on we neglect the issues related to deceptive idleness. Especially, since the state of B$\mathrm{WF}^{2} \mathrm{Q}+$ changes only in consequence of request arrivals and service, without losing generality, we assume that idling periods have zero duration, and we say that an application is backlogged iff its request queue inside $\mathrm{B}-\mathrm{WF}^{2} \mathrm{Q}+$ is not empty.

Given a budget $B=B_{i}^{j}$ assigned to an application, we denote as $R(B)$ the batch served using $B$. If an application becomes idle before consuming all of its budget $B$, or if it is deactivated because the remaining budget is lower than the size of the next request to serve, then the size of the batch $R(B)$ is lower than $B$. In this case we say that the budget is underutilized.

Suppose that only a portion $B^{\prime}<B$ of an underutilized budget is used by an application. According to the code in Fig. 4, three points can be highlighted. First, the virtual finish time of the batch is higher than it would have been if computed as a function of $B^{\prime}$. Hence the start of the service of $R(B)$ may be delayed. Second, this possibly delayed start, plus the fact that the disk is released in advance, are only beneficial for the completion time of the requests of the other applications. Third, when the application is done using $B$, its virtual finish time is properly decreased, so as to account only for the actual service received. Hence the next virtual start time will be correctly computed. In the end, the first issue is the only one to be addressed in computing worst-case guarantees. In this respect, in the proof of Lemma 2, we start from a fictitious scenario where a longer batch $\bar{R}(B)$ is served instead of $R(B)$.

Given any quantity $A$ defined for the real system, we use the superscript notation $A^{I}$ to denote its counterpart in the ideal system. For example, we denote as $W_{i}^{I}(t)$ the amount of service received by the $i$-th application in the ideal system during $[0, t]$. By definition, $V(t)$ is equal to the normalized amount of service that would be guaranteed by the fluid system to a continuously backlogged application during $[0, t]$ with respect to its weight, i.e., $W_{i}^{I}(t) \geq \phi_{i} \cdot V(t)$ holds for any application $i$ continuously backlogged during $[0, t]$. Moreover, we denote as $V_{i}(t)$ the virtual time of the $i$-th application. While the application is backlogged, $\frac{d V_{i}(t)}{d t}=\frac{1}{\phi_{i}} \cdot \frac{d W_{i}^{I}(t)}{d t}$, i.e., the growth of $V_{i}(t)$ measures the normalized amount of service received by the application in the ideal system. On the contrary, while the application is idle, $V_{i}(t)$ is fictitiously increased as follows: $V_{i}(t) \leftarrow \max \left(V_{i}\left(t^{-}\right), V(t)\right)$. The ideal system serves applications in such a way to guarantee that $\forall i, t V_{i}(t) \geq V(t)$. Finally, differently from the real system, it serves the requests of each application in FIFO order.

We denote as $F_{i}(t)$ the virtual finish time of the last batch, of the $i$-th application, already completed in the real system at time $t$. Recall that the real system starts serving a new batch only if it is eligible. Hence, from the time-stamping rules in Fig. 4, it follows that

$$
F_{i}(t) \leq V(t)+\frac{B_{i, \max }}{\phi_{i}}
$$

The second of the following lemmas will be used as a building block in proving both theorems.
Lemma 1: Let $R\left(B^{1}\right), R\left(B^{2}\right), \ldots, R\left(B^{k}\right)$ be $k$ batches (possibly of different applications) consecutively served in the real system during a time interval $\left[t_{s}, t_{f}\right]\left(R\left(B^{1}\right)\right.$ starts at time $t_{s}$, while $R\left(B^{k}\right)$ is completed at time $\left.t_{f}\right)$. Suppose that: 1$)$ the related $k$ budgets are fully utilized, 2) the $k$ batches are completed in the real system in the same order as in the ideal system, 3) there is a time instant $t_{0} \leq t_{s}$ such that at time $t_{0}$ the ideal system has not yet served any sector of any of the $k$ batches. Then, denoted as $t_{f}^{I}$ the time instant at which all the $k$ batches are completed in the ideal system, $W\left(t_{f}\right)-W\left(t_{f}^{I}\right) \leq W\left(t_{0}, t_{s}\right)$. Especially, if $t_{s}=0$, we have that $W\left(t_{f}\right)-W\left(t_{f}^{I}\right) \leq 0$, and hence $t_{f} \leq t_{f}^{I}$.

Proof: Since the batches are completed in the same order in both systems, to finish $R\left(B^{k}\right)$, the ideal system must complete all the $k$ batches. In addition, the ideal system might serve other batches during $\left[t_{0}, t_{f}^{I}\right]$. Hence, considering also the total service property, $W\left(t_{0}, t_{f}^{I}\right) \geq \sum_{i=1}^{k} B^{k}=W\left(t_{s}, t_{f}\right)=W\left(t_{0}, t_{f}\right)-$ $W\left(t_{0}, t_{s}\right)$. This inequality allows the thesis to be proven as follows:

$$
\begin{array}{r}
W\left(t_{f}\right)-W\left(t_{f}^{I}\right)=W\left(t_{0}, t_{f}\right)-W\left(t_{0}, t_{f}^{I}\right) \leq \\
W\left(t_{0}, t_{f}\right)-W\left(t_{0}, t_{f}\right)+W\left(t_{0}, t_{s}\right)=W\left(t_{0}, t_{s}\right)
\end{array}
$$

Before the second lemma, we need to define a last function. We define as lag of the $i$-th application at time $t$, the quantity

$$
\operatorname{lag}_{i}(t) \equiv W_{i}^{I}(t)-W_{i}(t)
$$

Lemma 2: Let $R_{i}^{j}$ be the $j$-th request issued by the $i$-th application, and let $s_{i}^{j}$ and $c_{i}^{j}$ be its start and completion time. We have that

$$
\operatorname{lag}_{i}\left(s_{i}^{j}\right) \leq B_{\max }+L_{\max }
$$

Moreover, if $\forall t \in\left[t_{1}, c_{i}^{j}\right] \frac{d W(t)}{d t}=T_{a g g}$ and all the applications are continuously backlogged in the ideal system, then, defined $R_{i}^{m}$ as the last requests served in the real system among the ones arrived during $\left[a_{i}^{j}, a_{i}^{j}+T_{F I F O}\right)\left(\right.$ possibly $R_{i}^{m}=R_{i}^{j}$ ),

$$
c_{i}^{j} \leq c_{i}^{I, m}+\frac{B_{i}^{l}-L_{i}^{j}}{\phi_{i} T_{a g g}}+\frac{B_{\max }+L_{\max }}{T_{a g g}}
$$

where $B_{i}^{l}$ is the budget used to serve $R_{i}^{j}$.

Proof: For the moment, we assume that $V(t)$ is smoothly tracked, and we postpone to the end of the proof the evaluation of the consequences of the stepwise approximation of $V(t)$ (outlined in Subsection II-E). We proceed as follows. First we consider a fictitious batch $\bar{R}\left(B_{i}^{l}\right)$ in which a fake request $R_{\text {fake }}$ is added after the last request of the actual batch $R\left(B_{i}^{l}\right)$. We suppose that $R_{f a k e}$ is long enough to let the whole budget $B_{i}^{l}$ be consumed $\left(L_{\text {fake }}=B-\sum_{R_{i}^{j} \in R(B)} L_{i}^{j}\right)$. We consider what would have happened if the fictitious batch $\bar{R}\left(B_{i}^{l}\right)$ would have been served in place of $R\left(B_{i}^{l}\right)$. We define this scenario as the fictitious scenario, as opposed to the actual scenario. Then we compute an upper bound to $\operatorname{lag}_{i}\left(s_{i}^{j}\right)$ and to $c_{i}^{j}$ as a function of quantities computed for the fictitious scenario.

With regards to $c_{i}^{j}$, due to the C-LOOK/FIFO service order of the real system, in addition to the requests queued at time $a_{i}^{j-}$, the request $R_{i}^{j}$ may also wait for the service of the other requests arrived during $\left[a_{i}^{j}, a_{i}^{j}+T_{F I F O}\right)$. In contrast, the ideal system serves the requests of each application in FIFO order. Hence in the real system $R_{i}^{j}$ is served as if $c_{i}^{I, j}=c_{i}^{I, m}$. 
Given the value of a quantity $q$ for the actual scenario, we use the over-line notation $\bar{q}$ to refer to the corresponding value for the fictitious scenario. Hence, $\bar{c}_{i}^{l} \geq s_{i}^{j}$ and $\bar{c}_{i}^{I, l} \geq c_{i}^{I, j}$ are e.g., the finish time of $\bar{R}\left(B_{i}^{l}\right)$ in the real and in the ideal system for the fictitious scenario. Note that $\forall t \leq c_{i}^{j} \bar{W}(t)=W(t)$. Finally, let $D_{i}^{j}$ be the sum of the sizes of the requests in $R\left(B_{i}^{l}\right)$ served after $R_{i}^{j}$, plus the size $L_{i}^{j}$ of $R_{i}^{j}$ itself.

Since we are computing worst-case guarantees, without losing generality, we assume that all the batches served before $\bar{R} B_{i}^{l}$ are fully utilized. We consider two cases. First, all the batches served during $\left[0, \bar{c}_{i}^{l}\right]$ in the real system for the fictitious scenario are completed in the same order in which they are completed in the ideal system. In this case, from Lemma 1, and considering that $c_{i}^{I, j}=\bar{c}_{i}^{I, j}$ and all the applications are continuously backlogged during $\left[c_{i}^{I, j}, \bar{c}_{i}^{I, l}\right]$, we have that $c_{i}^{j} \leq \bar{c}_{i}^{l} \leq \bar{c}_{i}^{I, l} \leq c_{i}^{I, j}+\frac{B_{i}^{l}-L_{i}^{j}}{\phi_{i} T_{a g g}}$. Hence (8) holds. Moreover, considering that $s_{i}^{j} \leq \bar{c}_{i}^{I, l}$ it also follows that, for the actual scenario, $W_{i}^{I}\left(s_{i}^{j}\right) \leq W_{i}\left(s_{i}^{j}\right)+D_{i}^{j} \leq$ $W_{i}\left(s_{i}^{j}\right)+B_{\max }$. Hence, (7) holds too.

Second, some of the batches served before $\bar{R}\left(B_{i}^{l}\right)$ in the real system for the fictitious scenario are completed after $R\left(B_{i}^{l}\right)$ in the ideal system. Let $R\left(B^{m}\right)$ be the last of these batches, and suppose it starts to be served at time $t_{0}$ in the real system. Let $R\left(B^{m+1}\right)$, $R\left(B^{m+2}\right), \ldots, R\left(B^{m+k}\right)=\bar{R}\left(B_{i}^{l}\right)$, be the successive batches served in the real system. Since the ordering among virtual finish times is the same as among the completion times in the ideal system of the corresponding batches [14], the virtual finish time of these $k$ batches is lower than the virtual finish time of $R\left(B^{m}\right)$. Hence, for $\mathrm{B}-\mathrm{WF}^{2} \mathrm{Q}+$ to choose $R\left(B^{m}\right)$ instead of one them, all these $k$ batches have to be not yet eligible or not yet arrived at time $t_{0}$. In both cases, the ideal system has not provided any service to any of them at time $t_{0}$. We also assume that $s_{i}^{j} \geq \bar{c}_{i}^{I, l}$, since, if the thesis holds in this (sub)case, it trivially holds also in case $s_{i}^{j}<\bar{c}_{i}^{I, l}$. Hence, thanks to Lemma 1, in the fictitious scenario, $\bar{W}_{i}^{I}\left(\bar{s}_{i}^{I, j}, s_{i}^{j}\right)=\bar{W}_{i}^{I}\left(\bar{s}_{i}^{I, j}, \bar{c}_{i}^{I, l}\right)+\bar{W}_{i}^{I}\left(\bar{c}_{i}^{I, l}, s_{i}^{j}\right)=D_{i}^{j}+$ $\bar{W}_{i}^{I}\left(\bar{c}_{i}^{I, l}, s_{i}^{j}\right) \leq D_{i}^{j}+W\left(\bar{c}_{i}^{I, l}, \bar{c}_{i}^{l}\right)-D_{i}^{j} \leq D_{i}^{j}+B^{m}-D_{i}^{j}=B^{m} \leq$ $B_{\max }$. Besides, since $s_{i}^{j}=\bar{s}_{i}^{j}, W_{i}^{I}\left(s_{i}^{j}\right) \leq \bar{W}_{i}^{I}\left(s_{i}^{j}\right)$ (because also the additional fake request $R_{\text {fake }}$ may have been served in the fictitious scenario), $\bar{W}_{i}^{I}\left(\bar{s}_{i}^{I, j}\right)=\bar{W}_{i}\left(s_{i}^{j}\right)$, and $\bar{W}_{i}^{I}\left(s_{i}^{j}\right)=$ $\bar{W}_{i}^{I}\left(\bar{s}_{i}^{I, j}\right)+\bar{W}_{i}^{I}\left(\bar{s}_{i}^{I, j}, s_{i}^{j}\right)$, then $W_{i}^{I}\left(s_{i}^{j}\right) \leq W_{i}\left(s_{i}^{j}\right)+B_{\max }$ in the actual scenario. This proves (7).

To prove (8), suppose for a moment that the real system is able to immediately preempt $R\left(B^{m}\right)$ as any of the batches $R\left(B^{m+1}\right)$, $R\left(B^{m+2}\right), \ldots, R\left(B_{i}^{l}\right)$ becomes eligible. Thanks to the hypothesis of the applications being continuously backlogged, this ability would not affect the request arrival pattern, and hence the order in which these $k$ batches would be served in the ideal system. Hence $c_{i}^{I, j}$ would not change. Moreover, thanks to Lemma 1 and to the arguments used for the previous case, $c_{i}^{j} \leq c_{i}^{I, j}+\frac{B_{i}^{l}-L_{i}^{j}}{\phi_{i} T_{a g g}}$ would hold. Unfortunately, due to the impossibility of preempting $R\left(B^{m}\right)$, the real system starts serving the $j$ batches with a delay of at most $\frac{B_{\max }}{T_{a g g}}$ time units, hence $c_{i}^{j} \leq c_{i}^{I, j}+\frac{B_{i}^{l}-L_{i}^{j}}{\phi_{i} T_{a g g}}+\frac{B_{\max }}{T_{a g g}}$.

Finally, for the reasons explained in Subsection III-A, the stepwise approximation of $V(t)$ causes the additional worst-case $L_{\max }$ and $\frac{L_{\max }}{T_{a g g}}$ components, respectively.

\section{Proof of Theorem 1}

To prove the theorem, we first compute the maximum perapplication deviation (lead/lag) of the real system with respect to the ideal system.

Lemma 3: The following inequalities hold:

$$
\begin{array}{r}
\forall i \in\{1,2, \ldots, N\}, t \\
\min \left(\phi_{i}\left(V_{i}(t)-F_{i}(t)\right), 0\right) \leq \operatorname{lag}_{i}(t) \leq B_{\max }+L_{\max }
\end{array}
$$

Proof: Once granted access to the disk, and until budget exhaustion, an application receives an amount of service equal to the total amount of service provided by the system. Hence, thanks to the total service property, $\operatorname{lag}_{i}(t)$ cannot decrease while the $i$-th application is being served. This has two consequences.

First, $\exists s_{i}^{l}: \forall t \operatorname{lag}_{i}(t) \leq \operatorname{lag}_{i}\left(s_{i}^{l}\right)$, where $s_{i}^{l}$ is the start time of a batch $R\left(B_{i}^{l}\right)$. Hence the rightmost inequality in (9) follows from Lemma 2. Second, $\exists c_{i}^{l}: \forall t \operatorname{lag}_{i}(t) \geq \operatorname{lag}_{i}\left(c_{i}^{l}\right)$, where $c_{i}^{l}$ is the completion time of a batch $R\left(B_{i}^{l}\right)$. If $F_{i}\left(c_{i}^{l}\right) \leq V_{i}\left(c_{i}^{l}\right)$, then $\operatorname{lag}_{i}\left(c_{i}^{l}\right) \geq 0$ and the leftmost inequality in (9) holds.

To prove the thesis in case $F_{i}\left(c_{i}^{l}\right)>V_{i}\left(c_{i}^{l}\right)$, let $s_{i}^{l}$ and $s_{i}^{I, l}$ be, respectively, the time instants at which the budget starts to be served in the real and in the ideal system. We have that $W_{i}^{I}\left(s_{i}^{I, l}\right)=W_{i}\left(s_{i}^{l}\right)$. It follows that

$$
W_{i}^{I}\left(c_{i}^{l}\right)-W_{i}\left(c_{i}^{l}\right)=W_{i}^{I}\left(s_{i}^{I, l}, c_{i}^{l}\right)-W_{i}\left(s_{i}^{l}, c_{i}^{l}\right) .
$$

Since $F_{i}\left(c_{i}^{l}\right)>V_{i}\left(c_{i}^{l}\right)$, the ideal system is still serving the budget $R\left(B_{i}^{l}\right)$ at time $c_{i}^{l}$, which implies $W_{i}^{I}\left(s_{i}^{I, l}, c_{i}^{l}\right)=$ $\phi_{i}\left(V_{i}\left(c_{i}^{l}\right)-S_{i}^{l}\right)$, where $S_{i}^{l}$ is the virtual start time of the batch $R\left(B_{i}^{l}\right)$. On the other hand, $W_{i}\left(s_{i}^{l}, c_{i}^{l}\right) \leq \phi_{i}\left(F_{i}\left(c_{i}^{l}\right)-S_{i}^{l}\right)$. Substituting this inequality and the previous equality in (10), we get $W_{i}^{I}\left(c_{i}^{l}\right)-W_{i}\left(c_{i}^{l}\right) \geq \phi_{i}\left(V_{i}\left(c_{i}^{l}\right)-S_{i}^{l}\right)-\phi_{i}\left(F_{i}\left(c_{i}^{l}\right)-S_{i}^{l}\right)=$ $\phi_{i}\left(V_{i}\left(c_{i}^{l}\right)-F_{i}\left(c_{i}^{l}\right)\right)$, which proves the thesis.

We can now prove Theorem 1 .

Proof: Let $a_{i}^{j}$ be the arrival time of the request that is waiting to be completed at time $t_{1}$. From the timestamping rule in Fig. 4, line 8 , and from the fact that the $i$-th application is continuously backlogged, it follows that the virtual start time of its batches served during $\left[t_{1}, t_{2}\right]$ is the same as if all these batches arrived back-to-back. Moreover, the service provided by the real system to the $i$-th application does not depend on the arrival pattern of the requests served after the one that is pending at time $t_{2}$. Finally, for the $i$-th application to receive at least the minimum amount of service that the real system is claimed to guarantee during $\left[t_{1}, t_{2}\right]$, it must ask for such service, i.e., the sum of the sizes of the requests issued during $\left[t_{1}, t_{2}\right]$, plus $Q_{i}\left(a_{i}^{j-}\right)$, must be no lower than this amount of service. Thanks to these considerations, to simplify the proofs, and without losing generality, we assume that, starting from time $a_{i}^{j}$, the $i$-th application is continuously backlogged and issues all its next requests asynchronously and back-to-back.

Using Lemma 3, we can write

$$
\begin{aligned}
W_{i}\left(t_{1}, t_{2}\right) & = \\
W_{i}^{I}\left(t_{2}\right)-\operatorname{lag}_{i}\left(t_{2}\right)-\left(W_{i}^{I}\left(t_{1}\right)-\operatorname{lag}_{i}\left(t_{1}\right)\right) & \geq \\
W_{i}^{I}\left(t_{2}\right)-B_{\max }-L_{\max }-W_{i}^{I}\left(t_{1}\right)+ & \\
+\min \left(\phi_{i}\left(V_{i}\left(t_{1}\right)-F_{i}\left(t_{1}\right)\right), 0\right) & \geq \\
\phi_{i}\left(V\left(t_{2}\right)-V_{i}\left(t_{1}\right)\right)+\min \left(\phi_{i}\left(V_{i}\left(t_{1}\right)-F_{i}\left(t_{1}\right)\right), 0\right)+ & \\
-B_{\max }-L_{\max } &
\end{aligned}
$$


where the last inequality follows from the fact that, since the $i$ th application is necessarily continuously backlogged also in the ideal system, then $W_{i}^{I}\left(t_{1}, t_{2}\right) \geq \phi_{i}\left(V\left(t_{2}\right)-V_{i}\left(t_{1}\right)\right)$. For any set of values of the other quantities, the rightmost term is lower if $F_{i}\left(t_{1}\right)>V_{i}\left(t_{1}\right)$. Hence, thanks to (4)

$$
\begin{aligned}
W_{i}\left(t_{1}, t_{2}\right) & \geq \\
\phi_{i}\left(V\left(t_{2}\right)-V_{i}\left(t_{1}\right)+V_{i}\left(t_{1}\right)-F_{i}\left(t_{1}\right)\right)-B_{\max }-L_{\max } & = \\
\phi_{i}\left(V\left(t_{2}\right)-F_{i}\left(t_{1}\right)\right)-B_{\max }-L_{\max } & \geq \\
\phi_{i}\left(V\left(t_{2}\right)-V\left(t_{1}\right)-\frac{B_{i, \max }}{\phi_{i}}\right)-B_{\max }-L_{\max } & \geq \\
\phi_{i} W\left(t_{1}, t_{2}\right)-B_{i, \max }-B_{\max }-L_{\max } &
\end{aligned}
$$

Before concluding the proof, it is important to note that, as anticipated in Subsection III-B, the $B_{i, \max }$ component in the rightmost term is a consequence of the real system being in advance with respect to the ideal system in serving the $i$-th application at time $a_{i}^{j}$. On the contrary, this component is equal to 0 if $R_{i}^{j}$ may arrive only after the minimum completion time guaranteed by the ideal system to the previous request $R_{i}^{j-1}$.

\section{Proof of Theorem 2}

Proof: Let $c_{i}^{j}$ and $c_{i}^{I, m}$ be the completion time of $R_{i}^{j}$ in the real system and of $R_{i}^{m}$ in the ideal system, respectively. We have that:

$$
\begin{aligned}
W\left(t_{1}, c_{i}^{I, m}\right) \leq V\left(t_{1}, c_{i}^{I, m}\right)=V_{i}\left(t_{1}\right)-V_{i}\left(t_{1}\right)+V\left(t_{1}, c_{i}^{I, m}\right) & = \\
V_{i}\left(t_{1}\right)-V_{i}\left(t_{1}\right)+V\left(c_{i}^{I, m}\right)-V\left(t_{1}\right) & = \\
\left(V_{i}\left(t_{1}\right)-V\left(t_{1}\right)\right)+\left(V\left(c_{i}^{I, m}\right)-V_{i}\left(t_{1}\right)\right) & \leq \\
\left(V_{i}\left(t_{1}\right)-V\left(t_{1}\right)\right)+\left(V_{i}\left(c_{i}^{I, m}\right)-V_{i}\left(t_{1}\right)\right) & \leq \\
\left(V_{i}\left(t_{1}\right)-V\left(t_{1}\right)\right)+\frac{W_{i}\left(t_{1}, c_{i}^{I, m}\right)}{\phi_{i}} &
\end{aligned}
$$

where the last inequality follows from the fact that the $i$-th application is continuously backlogged (in the ideal system) during $\left[t_{1}, c_{i}^{I, m}\right]$.

Let $Q^{I}\left(a_{i}^{j-}\right)$ be the backlog of the $i$-th application in the ideal system at time $a_{i}^{j-}$. We have that $W_{i}^{I}\left(t_{1}, c_{i}^{I, m}\right) \leq Q^{I}\left(t_{1}^{-}\right)+$ $A_{i}\left(t_{1}, a_{i}^{j}+T_{F I F O}\right)$. Hence, since the ideal system has a constant throughput equal to $T_{a g g}$, we have that

$c_{i}^{I, m}-t_{1} \leq \frac{\phi_{i}\left(V_{i}\left(t_{1}\right)-V\left(t_{1}\right)\right)+Q^{I}\left(t_{1}^{-}\right)+A_{i}\left(t_{1}, a_{i}^{j}+T_{F I F O}\right)}{\phi_{i} T_{a g g}}$

As a first step to derive the thesis from the above inequality, we can consider that, thanks to Lemma $3, Q^{I}\left(t_{1}^{-}\right)=Q\left(t_{1}^{-}\right)-$ $\operatorname{lag}_{i}\left(t_{1}\right) \leq Q\left(t_{1}^{-}\right)-\min \left(\phi_{i}\left(V_{i}\left(t_{1}\right)-F_{i}\left(t_{1}\right)\right), 0\right)$. Substituting this inequality in (14), we get

$$
\begin{array}{r}
c_{i}^{I, m}-t_{1} \leq \frac{\phi_{i}\left(V_{i}\left(t_{1}\right)-V\left(t_{1}\right)\right)+Q\left(t_{1}^{-}\right)}{\phi_{i} T_{a g g}}+ \\
+\frac{-\min \left(\phi_{i}\left(V_{i}\left(t_{1}\right)-F_{i}\left(t_{1}\right)\right), 0\right)+A_{i}\left(t_{1}, a_{i}^{j}+T_{F I F O}\right)}{\phi_{i} T_{a g g}}
\end{array}
$$

For any set of values of the other quantities, the rightmost term is higher if $F_{i}\left(t_{1}\right) \geq V_{i}\left(t_{1}\right)$. Hence, thanks to (4),

$$
\begin{aligned}
c_{i}^{I, m}-t_{1} & \leq \\
\frac{\phi_{i}\left(V_{i}\left(t_{1}\right)-V\left(t_{1}\right)\right)+Q\left(t_{1}^{-}\right)-\phi_{i}\left(V_{i}\left(t_{1}\right)-F_{i}\left(t_{1}\right)\right)+A_{i}\left(t_{1}, a_{i}^{j}+T_{F I F O}\right)}{\phi_{i} T_{a g g}} & = \\
\frac{\phi_{i}\left(F_{i}\left(t_{1}\right)-V\left(t_{1}\right)\right)+Q\left(t_{1}^{-}\right)+A_{i}\left(t_{1}, a_{i}^{j}+T_{F I F O}\right)}{\phi_{i} T_{a g g}} & \leq \\
\frac{B_{i, \max }+Q\left(t_{1}^{-}\right)+A_{i}\left(t_{1}, a_{i}^{j}+T_{F I F O}\right)}{\phi_{i} T_{a g g}} &
\end{aligned}
$$

The thesis follows from considering that, thanks to Lemma $2, c_{i}^{j}-c_{i}^{I, m} \leq \frac{B_{i}^{l}-L_{i}^{j}}{\phi_{i} T_{a g g}}+\frac{B_{\max }+L_{\max }}{T_{a g g}}$. Regarding the $B_{i, \max }$ component, the same considerations about the arrival pattern reported at the end of the proof of Theorem 1 apply. 